\title{
MULTISUMMABILITY OF FORMAL SOLUTIONS OF INHOMOGENEOUS LINEAR PARTIAL DIFFERENTIAL EQUATIONS WITH CONSTANT COEFFICIENTS
}

\author{
SŁAWOMIR MICHALIK
}

\begin{abstract}
We consider the Cauchy problem for a general inhomogeneous linear partial differential equation with constant coefficients in two complex variables. We obtain necessary and sufficient conditions for the multisummability of formal solutions in terms of analytic continuation properties and growth estimates of some functions connected with the inhomogeneity. The results are presented in the general framework of $1 / p$-fractional equations.
\end{abstract}

\section{INTRODUCTION}

We study the initial value problem for a general inhomogeneous linear $1 / p$-partial differential equation with constant coefficients in two complex variables $t, z$

(1) $P\left(\partial_{t}^{1 / p}, \partial_{z}^{1 / p}\right) u(t, z)=\hat{f}(t, z), \quad\left(\partial_{t}^{1 / p}\right)^{n} u(0, z)=\varphi_{n}(z) \quad$ for $n=0, \ldots, m-1$,

where $p \in \mathbb{N}, P(\lambda, \xi)$ is a polynomial of degree $m$ with respect to $\lambda$, the inhomogeneity $\hat{f}(t, z)$ is a formal $t^{1 / p}$-power series and the Cauchy data $\varphi_{n}(z)$ are $1 / p$-analytic functions (i.e. the functions $z \mapsto \varphi_{n}\left(z^{p}\right)$ are analytic) in a complex neighbourhood of the origin. By the fractional $1 / p$-derivative we mean the linear operator on the space of $z^{1 / p}$-power series defined by

$$
\partial_{z}^{1 / p}\left(\sum_{n=0}^{\infty} \frac{u_{n} z^{n / p}}{\Gamma(1+n / p)}\right):=\sum_{n=0}^{\infty} \frac{u_{n+1} z^{n / p}}{\Gamma(1+n / p)} .
$$

For $p=1, \partial_{z}^{1 / p}$ is the usual derivative and then (1) is the initial value problem for inhomogeneous linear partial differential equation with constant coefficients, where the inhomogeneity $\hat{f}(t, z)$ is a formal power series with respect to $t$ and the Cauchy data $\varphi_{n}(z)$ are analytic functions in a complex neighbourhood of the origin.

The characterisation of summable solutions of homogeneous linear PDEs with constant coefficients was previously studied by Balser [1, 3, 4], Balser and Miyake [8], Ichinobe [10], Lutz, Miyake and Schäfke [15], Malek [16], Michalik [17, 19, 20] and Miyake [21]. The summability properties of formal solutions of inhomogeneous equations was also investigated. Let us mention the papers of Balser [5] and Michalik [18] about the inhomogeneous heat equation, Balser and Loday-Richaud [7] about the inhomogeneous heat equation with variable coefficients and the paper of Balser, Duval and Malek [6], which is devoted to the abstract inhomogeneous Cauchy problem. We also refer to Balser and Yoshino [9], where the Gevrey order

2010 Mathematics Subject Classification. 35C10, 35C15, 35E15, 35R11.

Key words and phrases. fractional linear PDEs with constant coefficients, formal power series, Borel summability, multisummability, Duhamel principle. 
of formal solutions of general inhomogeneous linear PDEs with constant coefficients is characterised.

Since $1 / p$-derivative is the Caputo fractional derivative for $1 / p$-analytic functions [13], our previous papers $[19,20]$ and the present one give a new insight into the theory of fractional partial differential equations. Our approach is based on formal $1 / p$-power series solutions, $1 / p$-analytic functions and their integral representations. On the other hand, the $\alpha$-analytic solutions of fractional ordinary differential equations was studied in $[12,14]$ and the kind of fractional version of Duhamel principle was obtained in [23]. The survey of last results about the fractional partial differential equations can be found in [11].

In the paper we construct the formal solution $\hat{u}(t, z)$ of $(1)$ and study its Gevrey asymptotic properties. The main result concerns a characterisation of multisummability of solutions $\hat{u}(t, z)$ in terms of inhomogeneity. We proceed as follows. After making a suitable transformation of $\hat{u}(t, z)$ and of $\hat{f}(t, z)$, we can assume that the Cauchy data vanish. We represent $P(\lambda, \xi)$ in the form

$$
P(\lambda, \xi)=P_{0}(\xi)\left(\lambda-\lambda_{1}(\xi)\right)^{m_{1}} \ldots\left(\lambda-\lambda_{l}(\xi)\right)^{m_{l}},
$$

where $P_{0}(\xi)$ is a polynomial and $\lambda_{1}(\xi), \ldots, \lambda_{l}(\xi)$ are characteristic roots of multiplicity $m_{1}, \ldots, m_{l}\left(m_{1}+\ldots+m_{l}=m\right)$. In general, the formal solution of (1) may be not uniquely defined. To avoid this inconvenience, we choose a formal series $\hat{g}(t, z)$ satisfying the equation $P_{0}\left(\partial_{z}^{1 / p}\right) \hat{g}(t, z)=\hat{f}(t, z)$ and we find the formal solution $\hat{u}(t, z)$ of $(1)$, which is uniquely determined by $\hat{g}(t, z)$. Next we show that $\hat{u}(t, z)$ satisfies

$$
\hat{u}(t, z)=\sum_{j=1}^{l} \sum_{k=1}^{m_{j}} \hat{u}_{j k}(t, z)
$$

with $\hat{u}_{j k}(t, z)$ being a formal solution of

$$
\left(\partial_{t}^{1 / p}-\lambda_{j}\left(\partial_{z}^{1 / p}\right)\right)^{k} u_{j k}(t, z)=\hat{g}_{j k}(t, z),
$$

where $\lambda_{j}\left(\partial_{z}^{1 / p}\right)$ is a kind of pseudodifferential operator introduced in our paper [20] with $\lambda_{j}(\xi)$ defined by $(2)$, and $\hat{g}_{j k}(t, z)$ is a formal series connected with $\hat{g}(t, z)$. We prove that the Gevrey order of $\hat{u}_{j k}(t, z)$ depends on the order $q_{j}$ of the pole of $\lambda_{j}(\xi)$ at infinity and on the Gevrey order of $\hat{g}(t, z)$. We also characterise the analytic continuation (for $q_{j}=1$ ) and summability (for $q_{j}>1$ ) of $\hat{u}_{j k}(t, z)$ in terms of $\hat{g}(t, z)$. Finally, we obtain the characterisation of multisummable solutions of (1) in terms of $\hat{g}(t, z)$.

In the paper we apply the similar tools to that introduced in our previous papers $[19,20]$. In particular, we use the fractional derivatives $\partial_{z}^{1 / p}$, the pseudodifferential operators $\lambda\left(\partial_{z}^{1 / p}\right)$ and the operators $B^{\alpha, \beta}$, which are related to the Borel transformations.

\section{Notation, Gevrey formal power series And Borel summability}

We use the following notation. The complex disc in $\mathbb{C}^{n}$ with a centre at the origin and a radius $r>0$ is denoted by $D_{r}^{n}:=\left\{z \in \mathbb{C}^{n}:|z|<r\right\}$. To simplify notation, we write $D_{r}$ instead of $D_{r}^{1}$. If the radius $r$ is not essential, then we write it $D^{n}$ (resp. D) for short. 
$A$ sector in a direction $d \in \mathbb{R}$ with an opening $\varepsilon>0$ in the universal covering space $\widetilde{\mathbb{C}}$ of $\mathbb{C} \backslash\{0\}$ is defined by

$$
S(d, \varepsilon):=\left\{z \in \widetilde{\mathbb{C}}: z=r e^{i \theta}, d-\varepsilon / 2<\theta<d+\varepsilon / 2, r>0\right\} .
$$

Moreover, if the value of opening angle $\varepsilon$ is not essential, then we write $S_{d}$ for short. We denote by $\hat{S}_{d}$ the set $S_{d} \cup D$.

By $\mathcal{O}(G)$ we understand the space of analytic functions on a domain $G \subseteq \mathbb{C}^{n}$. The Banach space of analytic functions on $D_{r}$, continuous on its closure and equipped with the norm $\|\varphi\|_{r}:=\max _{|z| \leq r}|\varphi(z)|$ is denoted by $\mathbb{E}(r)$.

The space of formal power series

$$
\hat{u}(t, z)=\sum_{j=0}^{\infty} u_{j}(z) t^{j} \quad \text { with } \quad u_{j}(z) \in \mathbb{E}(r)
$$

is denoted by $\mathbb{E}(r)[[t]]$. Moreover, we set $\mathbb{E}[[t]]:=\bigcup_{r>0} \mathbb{E}(r)[[t]]$.

In this section we also recall some definitions and fundamental facts about the Gevrey formal power series and Borel summability. For more details we refer the reader to [2].

Definition 1. A function $u(t, z) \in \mathcal{O}\left(S(d, \varepsilon) \times D_{r}\right)$ is of exponential growth of order at most $s>0$ as $t \rightarrow \infty$ in $S(d, \varepsilon)$ if and only if for any $r_{1} \in(0, r)$ and any $\varepsilon_{1} \in(0, \varepsilon)$ there exist $A, B<\infty$ such that

$$
\max _{|z| \leq r_{1}}|u(t, z)|<A e^{B|t|^{s}} \quad \text { for every } \quad t \in S\left(d, \varepsilon_{1}\right) .
$$

The space of such functions is denoted by $\mathcal{O}^{s}\left(S(d, \varepsilon) \times D_{r}\right)$. We also write $\mathcal{O}^{s}\left(\hat{S}_{d} \times\right.$ $D)$ for the space $\mathcal{O}^{s}\left(S_{d} \times D\right) \cap \mathcal{O}\left(\hat{S}_{d} \times D\right)$.

Analogously, a function $\varphi(z) \in \mathcal{O}(S(d, \varepsilon))$ is of exponential growth of order at most $s>0$ as $z \rightarrow \infty$ in $S(d, \varepsilon)$ if and only if for any $\varepsilon_{1} \in(0, \varepsilon)$ there exist $A, B<\infty$ such that

$$
|\varphi(z)|<A e^{B|z|^{s}} \quad \text { for every } \quad z \in S\left(d, \varepsilon_{1}\right) .
$$

The space of such functions is denoted by $\mathcal{O}^{s}(S(d, \varepsilon))$. We also set $\mathcal{O}^{s}\left(\hat{S}_{d}\right):=$ $\mathcal{O}^{s}\left(S_{d}\right) \cap \mathcal{O}\left(\hat{S}_{d}\right)$.

Definition 2. Let $k>0$. A formal power series

$$
\hat{u}(t, z):=\sum_{j=0}^{\infty} u_{j}(z) t^{j} \quad \text { with } \quad u_{j}(z) \in \mathbb{E}(r)
$$

is $1 / k$-Gevrey formal power series in $t$ if its coefficients satisfy

$$
\max _{|z| \leq r}\left|u_{j}(z)\right| \leq A B^{j} \Gamma(1+j / k) \quad \text { for } \quad j=0,1, \ldots
$$

with some positive constants $A$ and $B$.

The set of $1 / k$-Gevrey formal power series in $t$ over $\mathbb{E}(r)$ is denoted by $\mathbb{E}(r)[[t]]_{1 / k}$. We also set $\mathbb{E}[[t]]_{1 / k}:=\bigcup_{r>0} \mathbb{E}(r)[[t]]_{1 / k}$. 
Definition 3. Let $k>0$ and $d \in \mathbb{R}$. A formal series $\hat{u}(t, z) \in \mathbb{E}[[t]]_{1 / k}$ defined by (3) is called $k$-summable in a direction $d$ if and only if its $k$-Borel transform

$$
\tilde{v}(t, z):=\sum_{j=0}^{\infty} u_{j}(z) \frac{t^{j}}{\Gamma(1+j / k)} \in \mathcal{O}^{k}\left(\hat{S}_{d} \times D\right) .
$$

The $k$-sum of $\hat{u}(t, z)$ in the direction $d$ is represented by the Laplace transform of $\tilde{v}(t, z)$

$$
u^{\theta}(t, z):=\frac{1}{t^{k}} \int_{0}^{\infty(\theta)} e^{-(s / t)^{k}} \tilde{v}(s, z) d s^{k},
$$

where the integration is taken over any ray $e^{i \theta} \mathbb{R}_{+}:=\left\{r e^{i \theta}: r \geq 0\right\}$ with $\theta \in$ $(d-\varepsilon / 2, d+\varepsilon / 2)$.

For every $k>0$ and $d \in \mathbb{R}$, according to the general theory of moment summability (see Section 6.5 in [2]), a formal series (3) is $k$-summable in the direction $d$ if and only if the same holds for the series

$$
\sum_{j=0}^{\infty} u_{j}(z) \frac{j ! \Gamma(1+j / k)}{\Gamma(1+j(1+1 / k))} t^{j}
$$

Consequently, we obtain a characterisation of $k$-summability (analogous to Definition 3), if we replace the $k$-Borel transform by the modified $k$-Borel transform

$$
v(t, z):=\mathcal{B}^{k} \hat{u}(t, z):=\sum_{j=0}^{\infty} u_{j}(z) \frac{j ! t^{j}}{\Gamma(1+j(1+1 / k))}
$$

and the Laplace transform by the Ecalle acceleration operator

$$
u^{\theta}(t, z)=t^{-k /(1+k)} \int_{0}^{\infty(\theta)} v(s, z) C_{1+1 / k}\left((s / t)^{k /(1+k)}\right) d s^{k /(1+k)}
$$

with $\theta \in(d-\varepsilon, d+\varepsilon)$. Here integration is taken over the ray $e^{i \theta} \mathbb{R}_{+}$and $C_{1+1 / k}(\zeta)$ is defined by

$$
C_{1+1 / k}(\zeta):=\frac{1}{2 \pi i} \int_{\gamma} u^{-1 /(k+1)} e^{u-\zeta u^{k /(k+1)}} d u
$$

with a path of integration $\gamma$ as in the Hankel integral for the inverse gamma function (from $\infty$ along $\arg u=-\pi$ to some $u_{0}<0$, then on the circle $|u|=\left|u_{0}\right|$ to $\arg u=\pi$, and back to $\infty$ along this ray).

Hence the $k$-summability is characterised as follows

Proposition 1. Let $k>0$ and $d \in \mathbb{R}$. A formal series $\hat{u}(t, z)$ given by (3) is $k$-summable in a direction $d$ if and only if its modified $k$-Borel transform

$$
\mathcal{B}^{k} \hat{u}(t, z)=\sum_{j=0}^{\infty} u_{j}(z) \frac{j ! t^{j}}{\Gamma(1+j(1+1 / k))} \in \mathcal{O}^{k}\left(\hat{S}_{d} \times D\right) .
$$

We are now ready to define multisummability in some multidirection.

Definition 4. Let $k_{1}>\ldots>k_{n}>0$. We say that a real vector $\left(d_{1}, \ldots, d_{n}\right) \in \mathbb{R}^{n}$ is an admissible multidirection if and only if

$$
\left|d_{j}-d_{j-1}\right| \leq \pi\left(1 / k_{j}-1 / k_{j-1}\right) / 2 \quad \text { for } \quad j=2, \ldots, n .
$$

Let $\mathbf{k}=\left(k_{1}, \ldots, k_{n}\right) \in \mathbb{R}_{+}^{n}$ and let $\mathbf{d}=\left(d_{1}, \ldots, d_{n}\right) \in \mathbb{R}^{n}$ be an admissible multidirection. We say that a formal power series $\hat{u}(t, z)$ given by $(3)$ is $\mathbf{k}$-summable in 
the multidirection $\mathbf{d}$ if and only if $\hat{u}(t, z)=\hat{u}_{1}(t, z)+\ldots+\hat{u}_{n}(t, z)$, where $\hat{u}_{j}(t, z)$ is $k_{j}$-summable in the direction $d_{j}$ for $j=1, \ldots, n$.

\section{3. $\alpha$-Derivatives, $\alpha$-ANALYTiC FunCtions AND OPERATORs $B^{\alpha, \beta}$}

In this section, in a similar way to [20], we introduce some tools to study divergent solutions of linear partial differential equations. First, we define some kind of fractional derivatives $\partial_{z}^{\alpha}$ of the formal power series in $\mathbb{C}\left[\left[z^{\alpha}\right]\right]$. These operators are the natural generalisation of the derivative $\partial_{z}$ defined into the space $\mathbb{C}[[z]]$. Namely, we have

Definition 5. Let $\alpha \in \mathbb{Q}_{+}$. The linear operator on the space of formal power series $\partial_{z}^{\alpha}: \mathbb{C}\left[\left[z^{\alpha}\right]\right] \rightarrow \mathbb{C}\left[\left[z^{\alpha}\right]\right]$ defined by the formula

$$
\partial_{z}^{\alpha}\left(\sum_{n=0}^{\infty} \frac{u_{n}}{\Gamma(1+\alpha n)} z^{\alpha n}\right)=\sum_{n=0}^{\infty} \frac{u_{n+1}}{\Gamma(1+\alpha n)} z^{\alpha n}
$$

is called an $\alpha$-derivative.

The right-inversion operator $\partial_{z}^{-\alpha}: \mathbb{C}\left[\left[z^{\alpha}\right]\right] \rightarrow \mathbb{C}\left[\left[z^{\alpha}\right]\right]$ given by

$$
\partial_{z}^{-\alpha}\left(\sum_{n=0}^{\infty} \frac{u_{n}}{\Gamma(1+\alpha n)} z^{\alpha n}\right)=\sum_{n=1}^{\infty} \frac{u_{n-1}}{\Gamma(1+\alpha n)} z^{\alpha n}
$$

is called an $\alpha$-integral.

Remark 1. Observe that such defined $\alpha$-derivative satisfies

$$
\partial_{z}^{\alpha} z^{\alpha n}=\left\{\begin{array}{lll}
0 & \text { for } \quad n=0 \\
\frac{\Gamma(1+\alpha n)}{\Gamma(1+\alpha(n-1))} z^{\alpha(n-1)} & \text { for } \quad n=1,2,3, \ldots
\end{array}\right.
$$

The same property has the Caputo derivative, which is defined (see (2.4.15) and (2.4.14) in [13]) by

$$
{ }^{C} D_{0+}^{\alpha} \varphi(z):=\frac{1}{\Gamma(n-\alpha)} \int_{0}^{z} \frac{\varphi^{(n)}(t) d t}{(z-t)^{\alpha-n-1}} \quad \text { for } \quad \alpha \notin \mathbb{N}_{0}, n \in \mathbb{N}, n-1<\alpha<n
$$

and ${ }^{C} D_{0+}^{n} \varphi(z):=\varphi^{(n)}(z)$ for $n \in \mathbb{N}$. Precisely speaking, the Caputo derivative satisfies (see Property 2.16 and (2.1.17) in [13])

$$
{ }^{C} D_{0+}^{\alpha} z^{\beta}=\frac{\Gamma(1+\beta)}{\Gamma(1+\beta-\alpha)} z^{\beta-\alpha}, \quad{ }^{C} D_{0+}^{\alpha} z^{k}=0 \quad \text { for } k=0, \ldots, n-1,
$$

where $n-1<\alpha<n, \beta>n-1$, and $n \in \mathbb{N}$.

Analogously, the $\alpha$-integral satisfies $\partial_{z}^{-\alpha} z^{\alpha n}=\frac{\Gamma(1+\alpha n)}{\Gamma(1+\alpha(n+1))} z^{\alpha(n+1)}$. The same property has the Riemann-Liouville fractional integral defined (see (2.2.1) in [13]) by

$$
I_{0+}^{\alpha} \varphi(z):=\frac{1}{\Gamma(\alpha)} \int_{0}^{z} \frac{\varphi(t) d t}{(z-t)^{1-\alpha}},
$$

which satisfies (see (2.2.10) in [13]) for $\beta>-1$ :

$$
I_{0+}^{\alpha} z^{\beta}=\frac{\Gamma(1+\beta)}{\Gamma(1+\alpha+\beta)} z^{\alpha+\beta} .
$$

We have 
Proposition 2. If $\hat{\varphi}(z) \in \mathbb{C}\left[\left[z^{\alpha}\right]\right]$ and $m \in \mathbb{N}$ then

$$
\left(\partial_{z}^{-\alpha}\right)^{m} \hat{\varphi}(z)=\partial_{z} \int_{0}^{z} \frac{(z-s)^{m \alpha}}{\Gamma(1+m \alpha)} \hat{\varphi}(s) d s .
$$

Proof. Let $\hat{\varphi}(z)=\sum_{n=0}^{\infty} \frac{\varphi_{n}}{\Gamma(1+n \alpha)} z^{n \alpha}$. Then the right-hand side of (5) is equal to

$$
\mathrm{RHS}=\partial_{z} \sum_{n=0}^{\infty} \frac{\varphi_{n}}{\Gamma(1+n \alpha) \Gamma(1+m \alpha)} \int_{0}^{z}(z-s)^{m \alpha} s^{n \alpha} d s .
$$

Moreover, using the beta integral we have

$$
\begin{aligned}
& \int_{0}^{z}(z-s)^{m \alpha} s^{n \alpha} d s=z^{(m+n) \alpha+1} \int_{0}^{1}(1-t)^{m \alpha} t^{n \alpha} d t \\
& =z^{(m+n) \alpha+1} B(1+m \alpha, 1+n \alpha)=z^{(m+n) \alpha+1} \frac{\Gamma(1+m \alpha) \Gamma(1+n \alpha)}{\Gamma(2+(m+n) \alpha)} .
\end{aligned}
$$

Hence

$$
\begin{aligned}
\text { RHS } & =\partial_{z} \sum_{n=0}^{\infty} \frac{\varphi_{n}}{\Gamma(2+(m+n) \alpha)} z^{(m+n) \alpha+1}=\sum_{n=0}^{\infty} \frac{\varphi_{n}}{\Gamma(1+(m+n) \alpha)} z^{(m+n) \alpha} \\
& =\sum_{n=m}^{\infty} \frac{\varphi_{n-m}}{\Gamma(1+n \alpha)} z^{n \alpha}=\text { LHS. }
\end{aligned}
$$

Definition 6. We say that a function $u(z)$ is $\alpha$-analytic on $G \subseteq \mathbb{C}$ (or, generally, on $G \subseteq \mathbb{C}^{n}$ ), where $G$ is a complex neighbourhood of the origin, if and only if the function $\zeta \mapsto u\left(\zeta^{1 / \alpha}\right)$ is analytic for every $z=\zeta^{1 / \alpha} \in G$. The space of $\alpha$-analytic functions on $G$ is denoted by $\mathcal{O}_{\alpha}(G)$.

We also say that a function $u(t, z)$ is $(\alpha, \beta)$-analytic on $G_{1} \times G_{2} \subseteq \mathbb{C}^{2}$ if and only if the function $(\tau, \zeta) \mapsto u\left(\tau^{1 / \alpha}, \zeta^{1 / \beta}\right)$ is analytic for every $(t, z)=\left(\tau^{1 / \alpha}, \zeta^{1 / \beta}\right) \in G_{1} \times$ $G_{2}$. The space of $(\alpha, \beta)$-analytic functions on $G_{1} \times G_{2}$ is denoted by $\mathcal{O}_{\alpha, \beta}\left(G_{1} \times G_{2}\right)$.

Moreover, analogously to Definition 1 , we denote by $\mathcal{O}_{\alpha}^{s}\left(\hat{S}_{d}\right)$ (resp. by $\mathcal{O}_{\alpha, \beta}^{s}\left(\hat{S}_{d_{1}} \times\right.$ $\left.\hat{S}_{d_{2}}\right)$ ) the space of $\alpha$-analytic functions on $\hat{S}_{d}$ (resp. the space of $(\alpha, \beta)$-analytic functions on $\hat{S}_{d_{1}} \times \hat{S}_{d_{2}}$ ) of exponential growth of order at most $s$ there.

If the formal power series $\hat{u}(z) \in \mathbb{C}\left[\left[z^{\alpha}\right]\right]$ is convergent in a complex neighbourhood of the origin, then its sum $u(z)$ is the $\alpha$-analytic function near the origin. For such functions we have well defined $\alpha$-derivative given by (4), which coincides with the Caputo fractional derivative (see Remark 1).

We also define the $\alpha$-Taylor series of $u(z) \in \mathcal{O}_{\alpha}(D)$ by the formula

$$
u(z)=\sum_{n=0}^{\infty} \frac{\left(\partial_{z}^{\alpha}\right)^{n} u(0)}{\Gamma(1+\alpha n)} z^{\alpha n} .
$$

Remark 2. For any $z_{0} \in \mathbb{C}$ we define the $\alpha$-derivative on the space $\mathbb{C}\left[\left[\left(z-z_{0}\right)^{\alpha}\right]\right]$ by the formula

$$
\partial_{z-z_{0}}^{\alpha}\left(\sum_{n=0}^{\infty} \frac{u_{n}}{\Gamma(1+\alpha n)}\left(z-z_{0}\right)^{\alpha n}\right)=\sum_{n=0}^{\infty} \frac{u_{n+1}}{\Gamma(1+\alpha n)}\left(z-z_{0}\right)^{\alpha n} .
$$


If the formal power series $\hat{u}(z) \in \mathbb{C}\left[\left[\left(z-z_{0}\right)^{\alpha}\right]\right]$ is convergent in a neighbourhood $G$ of $z_{0}$, then its sum $u(z)$ is the $\alpha$-analytic function with respect to $z_{0}$ on $G$. The $\alpha$-Taylor series of $u(z)$ with respect to $z_{0}$ is given by

$$
u(z)=\sum_{n=0}^{\infty} \frac{\left(\partial_{z-z_{0}}^{\alpha}\right)^{n} u\left(z_{0}\right)}{\Gamma(1+\alpha n)}\left(z-z_{0}\right)^{\alpha n} .
$$

Definition 7. The $\alpha$-exponentional function $e_{\alpha}(z)$ is defined by

$$
e_{\alpha}(z):=E_{\alpha}\left(z^{\alpha}\right)=\sum_{n=0}^{\infty} \frac{z^{\alpha n}}{\Gamma(1+\alpha n)},
$$

where $E_{\alpha}(z)$ denotes the Mittag-Leffler function.

The function $e_{\alpha}(z)$ plays the role of the exponential function $e^{z}$ in the case of $\alpha$-analytic functions. By the definition of $e_{\alpha}(z)$ and by the results on the MittagLeffler function (see [22]), we have

Proposition 3. The function $e_{\alpha}(z)$ satisfies the following properties:

a) $e_{\alpha}(z) \in \mathcal{O}_{\alpha}(\mathbb{C})$ and there exists $C<\infty$ such that $\left|e_{\alpha}(z)\right| \leq C e^{|z|}$ for every $z \in \mathbb{C}$

b) for every $a \in \mathbb{C}$ we have $\partial_{z}^{\alpha} e_{\alpha}(a z)=a^{\alpha} e_{\alpha}(a z)$,

c) if $\alpha<2$ and $\arg z \in(\pi / 2,2 \pi / \alpha-\pi / 2)$ then $e_{\alpha}(z) \rightarrow 0$ as $z \rightarrow \infty$.

Remark 3. Observe that $e_{\alpha}(z)$ satisfies

$$
\left\{\begin{array}{l}
\left(\partial_{z}^{\alpha}-1\right) e_{\alpha}(z)=0 \\
e_{\alpha}(0)=1
\end{array}\right.
$$

More generally, for every $m \in \mathbb{N}$ we define the function $e_{\alpha, m}(z)$ as the solution of the Cauchy problem

$$
\left\{\begin{array}{l}
\left(\partial_{z}^{\alpha}-1\right)^{m} e_{\alpha, m}(z)=0 \\
\left(\partial_{z}^{\alpha}\right)^{j} e_{\alpha, m}(0)=0 \\
\left(\partial_{z}^{\alpha}\right)^{m-1} e_{\alpha, m}(0)=1 .
\end{array} \text { for } \quad j=0, \ldots, m-2\right.
$$

It means that

(6)

$$
e_{\alpha, m}(z)=\sum_{n=0}^{\infty} \frac{n(n-1) \cdot \ldots \cdot(n-m+2)}{(m-1) !} \frac{z^{\alpha n}}{\Gamma(1+\alpha n)}=\sum_{n=m-1}^{\infty}\left(\begin{array}{c}
n \\
m-1
\end{array}\right) \frac{z^{\alpha n}}{\Gamma(1+\alpha n)} .
$$

In a similar way to $e_{\alpha}(z)$, the function $e_{\alpha, m}(z)$ satisfies the properties a) and c) of Proposition 3.

Since $\alpha \in \mathbb{Q}_{+}$and every $q / p$-analytic function is also $1 / p$-analytic, without loss of generality we may take $\alpha=1 / p$, where $p \in \mathbb{N}$. Observe that $1 / p$-analytic function is in fact an analytic function defined on the Riemann surface of $\sqrt[p]{z}$. Hence we have the following integral representation

Proposition 4 (see Lemma 1 in [20]). Let $\varphi(z) \in \mathcal{O}_{1 / p}\left(D_{r}\right)$. Then for every $|z|<\varepsilon<r$ and $k \in \mathbb{N}$ we have

$$
\left(\partial_{z}^{1 / p}\right)^{k} \varphi(z)=\frac{1}{2 p \pi i} \oint_{|w|=\varepsilon}^{p} \varphi(w) \int_{0}^{\infty(\theta)} \zeta^{k / p} e_{1 / p}(z \zeta) e^{-w \zeta} d \zeta d w
$$


for $\theta \in(-\arg w-\pi / 2,-\arg w+\pi / 2)$, where $\oint_{|w|=\varepsilon}^{p}$ denotes that we integrate $p$ times around the positively oriented circle of radius $\varepsilon$.

Moreover, there exist $\varrho \in(0, r)$ and $A, B<\infty$ such that

$$
\sup _{|z|<\varrho}\left|\left(\partial_{z}^{1 / p}\right)^{k} \varphi(z)\right| \leq A B^{k / p} \Gamma(1+k / p) \quad \text { for } \quad k=0,1, \ldots
$$

The formula (7) motivates the introduction of some kind of pseudodifferential operators on the space of $1 / p$-analytic functions. To this end, let $q(\xi)$ be an analytic function for $|\xi|>\left|\zeta_{0}^{1 / p}\right|$ of polynomial growth at infinity. Following [20] we define

$$
q\left(\partial_{z}^{1 / p}\right) e_{1 / p}(z \zeta):=q\left(\zeta^{1 / p}\right) e_{1 / p}(z \zeta) .
$$

Hence for every $\varphi(z) \in \mathcal{O}_{1 / p}\left(D_{r}\right)$ we have

$$
q\left(\partial_{z}^{1 / p}\right) \varphi(z):=\frac{1}{2 p \pi i} \oint_{|w|=\varepsilon}^{p} \varphi(w) \int_{\zeta_{0}}^{\infty(\theta)} q\left(\zeta^{1 / p}\right) e_{1 / p}(z \zeta) e^{-w \zeta} d \zeta d w
$$

with $\theta \in(-\arg w-\pi / 2,-\arg w+\pi / 2)$. Since $q(\xi)$ is a holomorphic function for $|\xi|>\left|\zeta_{0}^{1 / p}\right|$ and is of polynomial growth at infinity, the left-hand side of (8) is a well-defined $1 / p$-analytic function in a complex neighbourhood of the origin.

Now we introduce the operators $B^{\alpha, \beta}$, which are related to the modified $k$-Borel operators $\mathcal{B}^{k}$. Using the operators $B^{\alpha, \beta}$ we reduce the question about summability to the study of the solution of the appropriate Kowalevskaya type equation.

Definition 8. Let $\alpha, \beta \in \mathbb{Q}_{+}$. We define a linear operator on the space of formal power series

by the formula

$$
B^{\alpha, \beta}: \mathbb{E}\left[\left[t^{\alpha}\right]\right] \rightarrow \mathbb{E}\left[\left[t^{\beta}\right]\right]
$$

$$
B^{\alpha, \beta}(\hat{u}(t, z))=B^{\alpha, \beta}\left(\sum_{n=0}^{\infty} \frac{u_{n}(z)}{\Gamma(1+\alpha n)} t^{\alpha n}\right):=\sum_{n=0}^{\infty} \frac{u_{n}(z)}{\Gamma(1+\beta n)} t^{\beta n} .
$$

Observe that for any formal series $\hat{u}(t, z) \in \mathbb{E}[[t]]$ and $\mu, \nu \in \mathbb{N}, \mu>\nu$, we get

$$
\mathcal{B}^{k} \hat{u}(t, z)=\left(B^{1, \mu / \nu} \hat{u}\right)\left(t^{\nu / \mu}, z\right) \quad \text { with } \quad \mu / \nu=1+1 / k .
$$

Hence for $k \in \mathbb{Q}_{+}$we reformulate Proposition 1 as follows

Proposition 5. Let $\mu, \nu \in \mathbb{N}, \mu>\nu, k=(\mu / \nu-1)^{-1}$. Then the formal series $\hat{u}(t, z) \in \mathbb{E}[[t]]$ is $k$-summable in a direction $d$ if and only if

$$
B^{1, \mu / \nu} \hat{u}(t, z) \in \mathcal{O}_{\mu / \nu, 1}^{k+1}\left(\hat{S}_{(d+2 j \pi) \nu / \mu} \times D\right) \quad \text { for } \quad j=0, \ldots, \mu-1 .
$$

We recall the important properties of the operators $B^{\alpha, \beta}$, which play the crucial role in our study of summability. Namely, immediately from the definition we have

Proposition 6 (see also Proposition 4 in $[20]$ ). Let $\alpha, \beta \in \mathbb{Q}_{+}$and $\hat{u}(t, z) \in \mathbb{E}\left[\left[t^{\alpha}\right]\right]$. Then operator $B^{\alpha, \beta}$ and derivatives satisfy the following commutation formulas:

a) $B^{\alpha, \beta} \partial_{t}^{\alpha} \hat{u}(t, z)=\partial_{t}^{\beta} B^{\alpha, \beta} \hat{u}(t, z)$,

b) $B^{\alpha, \beta} \partial_{z} \hat{u}(t, z)=\partial_{z} B^{\alpha, \beta} \hat{u}(t, z)$,

c) $B^{\alpha, \beta} P\left(\partial_{t}^{\alpha}, \partial_{z}\right) \hat{u}(t, z)=P\left(\partial_{t}^{\beta}, \partial_{z}\right) B^{\alpha, \beta} \hat{u}(t, z)$ for any polynomial $P(\lambda, \xi)$ with constant coefficients.

At the end of this section, following [20], we extend the notion of Gevrey orders and Borel summability to formal power series in $t^{1 / p}$. 
Definition 9. Let $\gamma \in \mathbb{Q}_{+}$. The Banach space of $\gamma$-analytic functions on $D_{r}$, continuous on its closure and equipped with the norm $\|\varphi\|_{r}:=\max _{|z| \leq r}|\varphi(z)|$ is denoted by $\mathbb{E}_{\gamma}(r)$.

Definition 10. Let $k>0$ and $\gamma \in \mathbb{Q}_{+}$. A formal power series

$$
\hat{u}(t, z):=\sum_{j=0}^{\infty} u_{j}(z) t^{j / p} \quad \text { with } \quad u_{j}(z) \in \mathbb{E}_{\gamma}(r)
$$

is $1 / k$-Gevrey formal power series in $t^{1 / p}$ if its coefficients satisfy

$$
\max _{|z| \leq r}\left|u_{j}(z)\right| \leq A B^{j / p} \Gamma(1+j / k p) \quad \text { for } \quad j=0,1, \ldots
$$

with some positive constants $A$ and $B$.

The set of $1 / k$-Gevrey formal power series in $t^{1 / p}$ over $\mathbb{E}_{\gamma}(r)$ is denoted by $\mathbb{E}_{\gamma}(r)\left[\left[t^{1 / p}\right]\right]_{1 / k}$. We also set $\mathbb{E}_{\gamma}\left[\left[t^{1 / p}\right]\right]_{1 / k}:=\bigcup_{r>0} \mathbb{E}_{\gamma}(r)\left[\left[t^{1 / p}\right]\right]_{1 / k}$.

Definition 11. Let $k>0$ and $d \in \mathbb{R}$. A formal series $\hat{u}(t, z) \in \mathbb{E}_{\gamma}\left[\left[t^{1 / p}\right]\right]_{1 / k}$ is called $k$-summable in a direction $d$ if and only if the series $\hat{w}(t, z):=\hat{u}\left(t^{p}, z\right)$ is $k p$-summable in a direction $d / p$.

Similarly to Proposition 5 , we have the following characterisation of $k$-summability by the operators $B^{\alpha, \beta}$

Proposition 7 (see Proposition 7 in [20]). Let $\mu, \nu \in \mathbb{N}, \mu>\nu, k=(\mu / \nu-1)^{-1}$ and $d \in \mathbb{R}$. The formal series $\hat{u}(t, z) \in \mathbb{E}_{1 / p}\left[\left[t^{1 / p}\right]\right]$ is $k$-summable in a direction $d$ if and only if

$$
B^{1 / p, \mu / \nu p} \hat{u}(t, z) \in \mathcal{O}_{\mu / \nu p, 1 / p}^{k+1}\left(\hat{S}_{(d+2 j \pi) \nu / \mu} \times D\right) \quad \text { for } \quad j=0, \ldots, \mu-1 .
$$

\section{THE FORMAL SOLUTION}

In this section we find the formal solution of inhomogeneous fractional linear PDE and we prove the version of Duhamel principle for fractional equations. We also show that the solution of general inhomogeneous fractional equation is equal to the sum of simple inhomogeneous pseudodifferential equations.

Let us consider the Cauchy problem for general inhomogeneous fractional linear PDE with constant coefficients

$$
\left\{\begin{array}{l}
P\left(\partial_{t}^{1 / p}, \partial_{z}^{1 / p}\right) \tilde{u}(t, z)=\hat{\tilde{f}}(t, z) \\
\left(\partial_{t}^{1 / p}\right)^{n} \tilde{u}(0, z)=\varphi_{n}(z) \in \mathcal{O}_{1 / p}(D) \quad \text { for } \quad n=0, \ldots, m-1,
\end{array}\right.
$$

where $\hat{\tilde{f}}(t, z) \in \mathbb{E}_{1 / p}\left[\left[t^{1 / p}\right]\right]$ and $P(\lambda, \xi)$ is a polynomial of degree $m \in \mathbb{N}$ with respect to $\lambda$. In other words

$$
P(\lambda, \xi)=P_{0}(\xi) \lambda^{m}-\sum_{j=1}^{m} P_{j}(\xi) \lambda^{m-j}=P_{0}(\xi)\left(\lambda^{m}-\sum_{j=1}^{m} \tilde{P}_{j}(\xi) \lambda^{m-j}\right),
$$

where $P_{0}(\xi), \ldots, P_{m}(\xi)$ are polynomials and $\tilde{P}_{j}(\xi):=P_{j}(\xi) / P_{0}(\xi)(j=1, \ldots, m)$ are rational functions. 
Without loss of generality we may assume that the Cauchy data $\varphi_{n}(z)$ vanish. Indeed, after substitution

$$
u(t, z):=\tilde{u}(t, z)-\sum_{n=0}^{m-1} \frac{\varphi_{n}(z)}{\Gamma(1+n / p)} t^{n / p}
$$

we reduce the Cauchy problem (9) to

$$
\left\{\begin{array}{l}
P\left(\partial_{t}^{1 / p}, \partial_{z}^{1 / p}\right) u(t, z)=\hat{f}(t, z) \\
\left(\partial_{t}^{1 / p}\right)^{n} u(0, z)=0
\end{array} \quad \text { for } \quad n=0, \ldots, m-1\right.
$$

where

$$
\hat{f}(t, z):=\hat{\tilde{f}}(t, z)-P\left(\partial_{t}^{1 / p}, \partial_{z}^{1 / p}\right) \sum_{n=0}^{m-1} \frac{\varphi_{n}(z)}{\Gamma(1+n / p)} t^{n / p} \in \mathbb{E}_{1 / p}\left[\left[t^{1 / p}\right]\right] .
$$

Using the pseudodifferential operators defined by (8) we have

$$
\begin{aligned}
P\left(\partial_{t}^{1 / p}, \partial_{z}^{1 / p}\right) & =P_{0}\left(\partial_{z}^{1 / p}\right)\left(\left(\partial_{t}^{1 / p}\right)^{m}-\sum_{j=1}^{m} \tilde{P}_{j}\left(\partial_{z}^{1 / p}\right)\left(\partial_{t}^{1 / p}\right)^{m-j}\right) \\
& =: \quad P_{0}\left(\partial_{z}^{1 / p}\right) \tilde{P}\left(\partial_{t}^{1 / p}, \partial_{z}^{1 / p}\right) .
\end{aligned}
$$

Observe that, if $P_{0}\left(\partial_{z}^{1 / p}\right) \neq$ const. then the Cauchy problem (11) is not uniquely determined. In the homogeneous case this problem was solving by the choice of so called normalised formal solution (see [3] and [19]). In the inhomogeneous case the formal solution is determined by the function $\hat{g}(t, z) \in \mathbb{E}_{1 / p}\left[\left[t^{1 / p}\right]\right]$ (see also [9]), which satisfies the equation

$$
P_{0}\left(\partial_{z}^{1 / p}\right) \hat{g}(t, z)=\hat{f}(t, z)
$$

For given $\hat{g}(t, z)$ there is exactly one formal solution $\hat{u}(t, z)$ of the Cauchy problem

$$
\left\{\begin{array}{l}
\tilde{P}\left(\partial_{t}^{1 / p}, \partial_{z}^{1 / p}\right) u(t, z)=\hat{g}(t, z) \\
\left(\partial_{t}^{1 / p}\right)^{n} u(0, z)=0
\end{array} \quad \text { for } n=0, \ldots, m-1\right.
$$

which is also a solution of (11) and is called the formal solution of (11) determined by $\hat{g}(t, z)$.

We have

Proposition 8. The formal solution of (11) determined by $\hat{g}(t, z)$ is given by

$$
\hat{u}(t, z)=\sum_{n=0}^{\infty}\left(\partial_{t}^{-1 / p}\right)^{n+m} q_{n}\left(\partial_{z}^{1 / p}\right) \hat{g}(t, z)
$$

where $q_{n}(\xi)$ is the solution of the difference equation

$$
q_{n}(\xi)=\sum_{j=1}^{m} \tilde{P}_{j}(\xi) q_{n-j}(\xi) \quad \text { for } \quad n \geq 1
$$

with the initial conditions $q_{0}(\xi)=1$ and $q_{-1}(\xi)=\ldots=q_{-m+1}(\xi)=0$.

Proof. Since $q_{n}(\xi)$ are rational functions, they are of polynomial growth at infinity and they are holomorphic for sufficient large $|\xi|$. Hence the pseudodifferential operators $q_{n}\left(\partial_{z}^{1 / p}\right)$ are well defined. 
To finish the proof, it is sufficient to show that the formal series given by (13) is a solution of (12). To this end observe that $\left(\partial_{t}^{1 / p}\right)^{n} \hat{u}(0, z)=0$ for $n=0, \ldots, m-1$ and

$$
\begin{aligned}
& \tilde{P}\left(\partial_{t}^{1 / p}, \partial_{z}^{1 / p}\right) \hat{u}(t, z) \\
& =\left(\partial_{z}^{1 / p}\right) \sum_{n=0}^{\infty}\left(\partial_{t}^{-1 / p}\right)^{n} q_{n}\left(\partial_{z}^{1 / p}\right) \hat{g}(t, z)-\sum_{j=1}^{m} \sum_{n=0}^{\infty}\left(\partial_{t}^{-1 / p}\right)^{n+j} \tilde{P}_{j}\left(\partial_{z}^{1 / p}\right) q_{n}\left(\partial_{z}^{1 / p}\right) \hat{g}(t, z) \\
& =\sum_{n=0}^{\infty}\left(\partial_{t}^{-1 / p}\right)^{n} q_{n}\left(\partial_{z}^{1 / p}\right) \hat{g}(t, z)-\sum_{n=1}^{\infty} \sum_{j=1}^{m}\left(\partial_{t}^{-1 / p}\right)^{n} \tilde{P}_{j}\left(\partial_{z}^{1 / p}\right) q_{n-j}\left(\partial_{z}^{1 / p}\right) \hat{g}(t, z) \\
& =\sum_{n=0}^{\infty}\left(\partial_{t}^{-1 / p}\right)^{n} q_{n}\left(\partial_{z}^{1 / p}\right) \hat{g}(t, z)-\sum_{n=1}^{\infty}\left(\partial_{t}^{-1 / p}\right)^{n} q_{n}\left(\partial_{z}^{1 / p}\right) \hat{g}(t, z) \\
& =q_{0}\left(\partial_{z}^{1 / p}\right) \hat{g}(t, z)=\hat{g}(t, z) .
\end{aligned}
$$

Now, we show the following version of Duhamel's principle.

Theorem 1. The formal power series $\hat{u}(t, z)$ is a solution of (11) determined by $\hat{g}(t, z)$ if and only if

$$
\hat{u}(t, z)=\left(\partial_{t}^{1 / p}\right)^{p-1} \int_{0}^{t} \hat{u}(t, z ; s) d s
$$

where $\hat{u}(t, z ; s)$ is a formal solution of homogeneous equation

$$
\left\{\begin{array}{l}
\tilde{P}\left(\partial_{t-s}^{1 / p}, \partial_{z}^{1 / p}\right) u(t, z ; s)=0 \\
\left.\left(\partial_{t-s}^{1 / p}\right)^{n} u(t, z ; s)\right|_{t=s}=0 \\
\left.\left(\partial_{t-s}^{1 / p}\right)^{m-1} u(t, z ; s)\right|_{t=s}=\hat{g}(s, z)
\end{array} \quad(n=0, \ldots, m-2)\right.
$$

for every $s \in[0, t]$.

Proof. By the 1/p-Taylor formula, the solution of (15) is given by

$$
\hat{u}(t, z ; s)=\sum_{n=0}^{\infty} \frac{(t-s)^{n / p}}{\Gamma(1+n / p)} \tilde{q}_{n}\left(\partial_{z}^{1 / p}\right) \hat{g}(s, z)
$$

where $\tilde{q}_{n}(\xi)$ satisfies the difference equation (14) for $n \geq m$ with the initial conditions $\tilde{q}_{0}(\xi)=\ldots=\tilde{q}_{m-2}(\xi)=0$ and $\tilde{q}_{m-1}(\xi)=1$. It means that

$$
\tilde{q}_{n}(\xi)=q_{n-m+1}(\xi) \text { for } n \geq 0,
$$


where $q_{n-m+1}(\xi)$ were defined in Proposition 8. Hence, by (16), (17) and Proposition 2, we have

$$
\begin{aligned}
& \int_{0}^{t} \hat{u}(t, z ; s) d s=\sum_{n=0}^{\infty} \tilde{q}_{n}\left(\partial_{z}^{1 / p}\right) \int_{0}^{t} \frac{(t-s)^{n / p}}{\Gamma(1+n / p)} \hat{g}(s, z) d s \\
& =\sum_{n=0}^{\infty} \tilde{q}_{n}\left(\partial_{z}^{1 / p}\right)\left(\partial_{t}^{-1 / p}\right)^{n+p} \hat{g}(t, z) \\
& =\sum_{n=m-1}^{\infty} q_{n-m+1}\left(\partial_{z}^{1 / p}\right)\left(\partial_{t}^{-1 / p}\right)^{n-m+1}\left(\partial_{t}^{-1 / p}\right)^{m-1+p} \hat{g}(t, z) \\
& =\sum_{n=0}^{\infty} q_{n}\left(\partial_{z}^{1 / p}\right)\left(\partial_{t}^{-1 / p}\right)^{n}\left(\partial_{t}^{-1 / p}\right)^{m-1+p} \hat{g}(t, z) .
\end{aligned}
$$

Finally, by Proposition 8, we have

$$
\left(\partial_{t}^{1 / p}\right)^{p-1} \int_{0}^{t} \hat{u}(t, z ; s) d s=\sum_{n=0}^{\infty} q_{n}\left(\partial_{z}^{1 / p}\right)\left(\partial_{t}^{-1 / p}\right)^{n}\left(\partial_{t}^{-1 / p}\right)^{m} \hat{g}(t, z)=\hat{u}(t, z) .
$$

Let $\lambda_{1}(\xi), \ldots, \lambda_{l}(\xi)$ be characteristic roots of $\lambda \mapsto P(\lambda, \xi)$ of multiplicity $m_{1}, \ldots, m_{l}$ $\left(m_{1}+\ldots+m_{l}=m\right)$, where $P(\lambda, \xi)$ is a polynomial defined by (10). It means that

$$
P(\lambda, \xi)=P_{0}(\xi) \tilde{P}(\lambda, \xi)=P_{0}(\xi)\left(\lambda-\lambda_{1}(\xi)\right)^{m_{1}} \ldots\left(\lambda-\lambda_{l}(\xi)\right)^{m_{l}}
$$

and $\lambda_{j}(\xi)$ are algebraic functions. Hence there exists $R<\infty$ such that the functions $\lambda_{j}(\xi)$ are holomorphic for $|\xi| \geq R$, at least in the multiple-valued sense. Moreover for every characteristic function $\lambda_{j}(\xi)$ there exist a pole order $q_{j} \in \mathbb{Q}$ and a leading term $\lambda_{j} \in \mathbb{C} \backslash\{0\}$ satisfying $\lim _{\xi \rightarrow \infty} \lambda_{j}(\xi) / \xi^{q_{j}}=\lambda_{j}$. Hence, as in (8), we have the pseudodifferential operator $\lambda_{j}\left(\partial_{z}^{1 / p}\right)$, which for every $\varphi(z) \in \mathcal{O}_{1 / p}\left(D_{r}\right)$ is defined by

$$
\lambda_{j}\left(\partial_{z}^{1 / p}\right) \varphi(z):=\frac{1}{2 p \pi i} \oint_{|w|=\varepsilon}^{p} \varphi(w) \int_{\zeta_{0}}^{\infty(\theta)} \lambda_{j}\left(\zeta^{1 / p}\right) e_{1 / p}(z \zeta) e^{-w \zeta} d \zeta d w
$$

where $\zeta_{0}=R^{p} e^{i \theta}, \theta \in(-\arg w-\pi / 2,-\arg w+\pi / 2)$ and $\varepsilon<r$. Observe that by (18) we can factor the operator $P\left(\partial_{t}^{1 / p}, \partial_{z}^{1 / p}\right)$ as

$$
P\left(\partial_{t}^{1 / p}, \partial_{z}^{1 / p}\right)=P_{0}\left(\partial_{z}^{1 / p}\right)\left(\partial_{t}^{1 / p}-\lambda_{1}\left(\partial_{z}^{1 / p}\right)\right)^{m_{1}} \ldots\left(\partial_{t}^{1 / p}-\lambda_{l}\left(\partial_{z}^{1 / p}\right)\right)^{m_{l}} .
$$

Now, using Proposition 8 and the factorisation of operator $\tilde{P}\left(\partial_{t}^{1 / p}, \partial_{z}^{1 / p}\right)$, we obtain the following decomposition of solution of (11) determined by $\hat{g}(t, z)$.

Theorem 2. Let $\hat{u}(t, z)$ be a formal solution of

$$
\tilde{P}\left(\partial_{t}^{1 / p}, \partial_{z}^{1 / p}\right) u(t, z)=\hat{g}(t, z), \quad\left(\partial_{t}^{1 / p}\right)^{n} u(0, z)=0 \quad n=0, \ldots, m-1,
$$

where

$$
\tilde{P}\left(\partial_{t}^{1 / p}, \partial_{z}^{1 / p}\right)=\left(\partial_{t}^{1 / p}-\lambda_{1}\left(\partial_{z}^{1 / p}\right)\right)^{m_{1}} \ldots\left(\partial_{t}^{1 / p}-\lambda_{l}\left(\partial_{z}^{1 / p}\right)\right)^{m_{l}} .
$$

Then $\hat{u}(t, z)=\sum_{j=1}^{l} \sum_{k=1}^{m_{j}} \hat{u}_{j k}(t, z)$, where $\hat{u}_{j k}(t, z)$ is a formal solution of

$$
\begin{cases}\left(\partial_{t}^{1 / p}-\lambda_{j}\left(\partial_{z}^{1 / p}\right)\right)^{k} u_{j k}(t, z)=\hat{g}_{j k}(t, z) & \\ \left(\partial_{t}^{1 / p}\right)^{n} u_{j k}(0, z)=0 & n=0, \ldots, k-1\end{cases}
$$


and $\hat{g}_{j k}(t, z)=d_{j k}\left(\partial_{z}^{1 / p}\right) \hat{g}(t, z)$ for some holomorphic functions $d_{j k}(\xi)$ of polynomial growth.

Proof. By Theorem 1, $\hat{u}(t, z)=\left(\partial_{t}^{1 / p}\right)^{p-1} \int_{0}^{t} \hat{u}(t, z ; s) d s$ and $\hat{u}(t, z ; s)$ is a solution of

$$
\left\{\begin{array}{l}
P\left(\partial_{t-s}^{1 / p}, \partial_{z}^{1 / p}\right) u(t, z ; s)=0 \\
\left.\left(\partial_{t-s}^{1 / p}\right)^{n} u(t, z ; s)\right|_{t=s}=0 \\
\left.\left(\partial_{t-s}^{1 / p}\right)^{m-1} u(t, z ; s)\right|_{t=s}=\hat{g}(s, z) .
\end{array} \quad \text { for } \quad n=0, \ldots, m-2\right.
$$

Let us recall that

$$
\hat{u}(t, z ; s)=\sum_{n=0}^{\infty} \frac{(t-s)^{n / p}}{\Gamma(1+n / p)} \tilde{q}_{n}\left(\partial_{z}^{1 / p}\right) \hat{g}(s, z),
$$

where the rational functions $\tilde{q}_{n}(\xi)$ satisfy

$$
\tilde{q}_{n}(\xi)=\sum_{j=1}^{m} \tilde{P}_{j}(\xi) \tilde{q}_{m-j}(\xi)(n \geq m), \quad \tilde{q}_{0}(\xi)=\ldots=\tilde{q}_{m-2}(\xi)=0, \quad \tilde{q}_{m-1}(\xi)=1 .
$$

On the other hand

$$
\tilde{q}_{n}(\xi)=\sum_{j=1}^{l} \sum_{k=0}^{m_{j}-1} c_{j k}(\xi) \frac{n !}{(n-k) !} \lambda_{j}^{n}(\xi),
$$

where $c_{j k}(\xi)$ are the holomorphic functions of polynomial growth for sufficiently large $|\xi|$ (see Section 5 in [19] for more details) and $\lambda_{j}(\xi)$ are characteristic roots of multiplicity $m_{j}$.

It means that

$$
\hat{u}(t, z ; s)=\left(\sum_{j=1}^{l} \sum_{k=0}^{m_{j}-1} c_{j k}\left(\partial_{z}^{1 / p}\right) \sum_{n=0}^{\infty} \frac{n !}{(n-k) !} \lambda_{j}^{n}\left(\partial_{z}^{1 / p}\right) \frac{(t-s)^{n / p}}{\Gamma(1+n / p)}\right) \hat{g}(s, z) .
$$

Hence

$$
\hat{u}(t, z ; s)=\sum_{j=1}^{l} \sum_{k=0}^{m_{j}-1} \hat{u}_{j k}(t, z ; s)
$$

and

$$
\hat{u}_{j k}(t, z ; s)=c_{j k}\left(\partial_{z}^{1 / p}\right) \sum_{n=0}^{\infty} \frac{n !}{(n-k) !} \lambda_{j}^{n}\left(\partial_{z}^{1 / p}\right) \frac{(t-s)^{n / p}}{\Gamma(1+n / p)} \hat{g}(s, z)
$$

satisfies the equation

$$
\left\{\begin{array}{l}
\left(\partial_{t-s}^{1 / p}-\lambda_{j}\left(\partial_{z}^{1 / p}\right)\right)^{k} u_{j k}(t, z ; s)=0 \\
\left.\left(\partial_{t-s}^{1 / p}\right)^{n} u_{j k}(t, z ; s)\right|_{t=s}=0 \\
\left.\left(\partial_{t-s}^{1 / p}\right)^{k} u_{j k}(t, z ; s)\right|_{t=s}=\hat{g}_{j k}(s, z),
\end{array} \text { for } \quad n=0, \ldots, k-1\right.
$$

where

$$
\hat{g}_{j k}(t, z)=d_{j k}\left(\partial_{z}^{1 / p}\right) \hat{g}(t, z) \quad \text { and } \quad d_{j k}(\xi)=k ! c_{j k}(\xi) \lambda_{j}^{k}(\xi) .
$$

Applying once again Theorem 1 we obtain the assertion. 
By the above theorem, it is sufficient to consider the equation

$$
\begin{cases}\left(\partial_{t}^{1 / p}-\lambda\left(\partial_{z}^{1 / p}\right)\right)^{k} u(t, z)=\hat{g}(t, z) & \\ \left(\partial_{t}^{1 / p}\right)^{n} u(0, z)=0, & n=0, \ldots, k-1\end{cases}
$$

We will investigate the behaviour of solution $\hat{u}(t, z)$ depends on the pole order $q$ of the characteristic root $\lambda(\xi)$.

- For $q=1$ we will study the analytic continuation property of $u(t, z)$ depends on $g(t, z)$.

- For $q>1$ we will study the summability property of $\hat{u}(t, z)$ depends on $\hat{g}(t, z)$.

\section{Gevrey estimates}

In this section we study the Gevrey order of formal solution to inhomogeneous equation. The similar result was proved by Balser and Yoshino [9].

Theorem 3. Let $\hat{u}(t, z)$ be a formal solution of (11) determined by a Gevrey series $\hat{g}(t, z) \in \mathbb{E}_{1 / p}\left[\left[t^{1 / p}\right]\right]_{s}$ of order $s>0$ and let $\hat{u}(t, z)=\sum_{j=1}^{l} \sum_{k=0}^{m_{j}-1} \hat{u}_{j k}(t, z)$ be $a$ decomposition of solution constructed in Theorem 2. Then $\hat{u}_{j k}(t, z)$ is a Gevrey series of order $\max \left\{q_{j}-1, s\right\}$ with respect to $t$, where $q_{j} \in \mathbb{Q}$ is a pole order of characteristic root $\lambda_{j}(\xi)$.

Proof. By Theorem 2, $\hat{u}_{j k}(t, z)$ is a formal solution of

$$
\left\{\begin{array}{l}
\left(\partial_{t}^{1 / p}-\lambda_{j}\left(\partial_{z}^{1 / p}\right)\right)^{k} u_{j k}(t, z)=\hat{g}_{j k}(t, z) \\
\left(\partial_{t}^{1 / p}\right)^{n} u_{j k}(0, z)=0 \text { for } n=0, \ldots, k-1
\end{array}\right.
$$

where $\hat{g}_{j k}(t, z)=d_{j k}\left(\partial_{z}^{1 / p}\right) \hat{g}(t, z)$. By Theorem 1, $\hat{u}_{j k}(t, z)=\left(\partial_{t}^{1 / p}\right)^{p-1} \int_{0}^{t} \hat{u}_{j k}(t, z ; s) d s$, where $\hat{u}_{j k}(t, z ; s)$ satisfies

$$
\left\{\begin{array}{l}
\left(\partial_{t}^{1 / p}-\lambda_{j}\left(\partial_{z}^{1 / p}\right)\right)^{k} u_{j k}(t, z ; s)=0 \\
\left.\left(\partial_{t}^{1 / p}\right)^{n} u(t, z ; s)\right|_{t=s}=0 \text { for } n=0, \ldots, k-2 \\
\left.\left(\partial_{t}^{1 / p}\right)^{k-1} u(t, z ; s)\right|_{t=s}=\hat{g}_{j k}(s, z)
\end{array}\right.
$$

If $\hat{g}(t, z)=\sum_{m=0}^{\infty} \frac{g_{m}(z)}{\Gamma(1+m / p)} t^{m / p}$ then $\hat{g}_{j k}(t, z)=\sum_{m=0}^{\infty} \frac{d_{j k}\left(\partial_{z}^{1 / p}\right) g_{m}(z)}{\Gamma(1+m / p)} t^{m / p}$. Hence

$$
\hat{u}_{j k}(t, z ; s)=\sum_{n=0}^{\infty} \sum_{m=0}^{\infty} \frac{(t-s)^{n / p} s^{m / p}}{\Gamma(1+n / p) \Gamma(1+m / p)} \frac{n !}{(n-k) !} \lambda^{n}\left(\partial_{z}^{1 / p}\right) d_{j k}\left(\partial_{z}^{1 / p}\right) g_{m}(z)
$$


and using the beta integral

$$
\begin{aligned}
& \hat{u}_{j k}(t, z)=\left(\partial_{t}^{1 / p}\right)^{p-1} \int_{0}^{t} \hat{u}_{j k}(t, z ; s) d s \\
& =\sum_{n=0}^{\infty} \sum_{m=0}^{\infty} \frac{n !}{(n-k) !} \lambda^{n}\left(\partial_{z}^{1 / p}\right) d_{j k}\left(\partial_{z}^{1 / p}\right) g_{m}(z)\left(\partial_{t}^{1 / p}\right)^{p-1} \int_{0}^{t} \frac{(t-s)^{n / p} s^{m / p}}{\Gamma(1+n / p) \Gamma(1+m / p)} d s \\
& =\sum_{n=0}^{\infty} \sum_{m=0}^{\infty} \frac{n !}{(n-k) !} \lambda^{n}\left(\partial_{z}^{1 / p}\right) d_{j k}\left(\partial_{z}^{1 / p}\right) g_{m}(z)\left(\partial_{t}^{1 / p}\right)^{p-1} \frac{t^{\frac{n+m}{p}+1}}{\Gamma\left(2+\frac{n+m}{p}\right)} \\
& =\sum_{n=0}^{\infty} \sum_{m=0}^{\infty} \frac{n !}{(n-k) !} \lambda^{n}\left(\partial_{z}^{1 / p}\right) d_{j k}\left(\partial_{z}^{1 / p}\right) g_{m}(z) \frac{t^{\frac{n+m+1}{p}}}{\Gamma\left(1+\frac{n+m+1}{p}\right)} \\
& =\sum_{l=1}^{\infty} \frac{t^{l / p}}{\Gamma(1+l / p)} \sum_{m=0}^{l-1} \frac{(l-m-1) !}{(l-m-1-k) !} \lambda^{l-m-1}\left(\partial_{z}^{1 / p}\right) d_{j k}\left(\partial_{z}^{1 / p}\right) g_{m}(z) \\
& =: \sum_{l=1}^{\infty} \frac{u_{l}(z) t^{l / p}}{\Gamma(1+l / p)} .
\end{aligned}
$$

Since $\hat{g}(t, z) \in \mathbb{E}_{1 / p}\left[\left[t^{1 / p}\right]\right]_{s}$, there exist $A, B<\infty$ and $\varrho>0$ such that

$$
\sup _{|z|<\varrho / 2}\left|g_{m}(z)\right| \leq A B^{m} \Gamma(1+m(s+1) / p) \quad \text { for } \quad m=0,1, \ldots
$$

Now, repeating the proof of Theorem 1 in [19], we obtain the estimate

$$
\begin{aligned}
\left|u_{l}(z)\right| & \leq \sum_{m=0}^{l-1} \frac{(l-m-1) !}{(l-m-1-k) !}\left|\lambda^{l-m-1}\left(\partial_{z}^{1 / p}\right) d_{j k}\left(\partial_{z}^{1 / p}\right) g_{m}(z)\right| \\
& \leq \tilde{A} \tilde{B}^{l} \sum_{m=0}^{l-1} \Gamma(1+(l-m-1) q / p) \Gamma(1+m(s+1) / p)
\end{aligned}
$$

for $z \in D_{\varrho / 2}$. Hence there exist $C, D<\infty$ such that

$$
\sup _{|z|<\varrho / 2}\left|u_{l}(z)\right| \leq C D^{l} \Gamma(1+l(\tilde{s}+1) / p) \quad \text { for } \quad l=0,1,2, \ldots,
$$

where $\tilde{s}=\max \{s, q-1\}$.

\section{Analytic solution}

In this section we study the solution of the Cauchy problem

$$
\begin{cases}\left(\partial_{t}^{1 / p}-\lambda\left(\partial_{z}^{1 / p}\right)\right)^{m} u(t, z)=g(t, z) & \\ \left(\partial_{t}^{1 / p}\right)^{n} u(0, z)=0, & n=0, \ldots, m-1,\end{cases}
$$

where $g(t, z) \in \mathcal{O}_{1 / p}\left(D^{2}\right), \lambda(\xi) \in \mathcal{O}\left(|\xi|>\left|\zeta_{0}^{1 / p}\right|\right)$ and $\lim _{\xi \rightarrow \infty} \lambda(\xi) / \xi=\lambda \in \mathbb{C} \backslash\{0\}$.

We would like to find the necessary and sufficient conditions for the analytic continuation property of solution

$$
u(t, z) \in \mathcal{O}_{1 / p}^{s}\left(\hat{S}_{d} \times D\right)
$$

in terms of inhomogeneity $g(t, z)$. 
Since $g(t, z)=\left(\partial_{t}^{1 / p}-\lambda\left(\partial_{z}^{1 / p}\right)\right)^{m} u(t, z)$, we immediately obtain the necessary condition for $(21)$

$$
u(t, z) \in \mathcal{O}_{1 / p}^{s}\left(\hat{S}_{d} \times D\right) \Longrightarrow g(t, z) \in \mathcal{O}_{1 / p}^{s}\left(\hat{S}_{d} \times D\right) .
$$

But this condition is too weak, as the following example shows. Let us consider the equation

$$
\partial_{t} u-\partial_{z} u=\frac{1}{(1-z)^{2}}, \quad u(0, z)=0
$$

Observe that the inhomogeneity satisfies $g(t, z)=\frac{1}{(1-z)^{2}} \in \mathcal{O}\left(\hat{S}_{0} \times D\right)$, but the solution $u(t, z)=\frac{1}{1-t-z}-\frac{1}{1-z}$ is not analytic on $\hat{S}_{0} \times D$.

To find the better characterisation of $(21)$ in terms of $g(t, z)$, we define the function

$G_{m}(g, \lambda(\cdot) ; t, z):=\int_{0}^{t} \oint_{|w|=\varepsilon}^{p} g(s, w) \int_{\zeta_{0}}^{\infty} e_{1 / p, m}\left((t-s) \lambda^{p}\left(\zeta^{1 / p}\right)\right) e_{1 / p}(z \zeta) e^{-w \zeta} d \zeta d w d s$,

where the function $e_{1 / p, m}(z)$ is given by $(6)$.

Deforming the path of integration with respect to $w$ in (22) and repeating the proof of Lemma 3 in [20] (see also Lemma 2 in [19]) we obtain

Lemma 1. Let $s>1$ and $g(t, z) \in \mathcal{O}_{1 / p}^{s}\left(\hat{S}_{d} \times \hat{S}_{d+p \arg \lambda}\right)$. Then also $G_{m}(g, \lambda(\cdot) ; t, z) \in$ $\mathcal{O}_{1 / p}^{s}\left(\hat{S}_{d} \times \hat{S}_{d+p \arg \lambda}\right)$.

We show the connection between the function $G_{m}(g, \lambda(\cdot) ; t, z)$ and the solution of the Cauchy problem (20)

Lemma 2. Let $u(t, z)$ be a solution of (20). Then the function $G_{m}(g, \lambda(\cdot) ; t, z)$ satisfies the following formulas

(a) $\lambda^{m-1}\left(\partial_{z}^{1 / p}\right) u(t, z)=\left(\partial_{t}^{1 / p}\right)^{p-1} \frac{1}{2 p \pi i} G_{m}(g, \lambda(\cdot) ; t, z)$,

(b) $\lambda^{k}\left(\partial_{z}^{1 / p}\right) G_{m-k}(g, \lambda(\cdot) ; t, z)=\left(\partial_{t}^{1 / p}-\lambda\left(\partial_{z}^{1 / p}\right)\right)^{k} G_{m}(g, \lambda(\cdot) ; t, z)$ for $k=0, \ldots, m-$ 1 ,

(c) $g(t, z)=\left(\partial_{t}^{1 / p}-\lambda\left(\partial_{z}^{1 / p}\right)\right)\left(\partial_{t}^{1 / p}\right)^{p-1} \frac{1}{2 p \pi i} G_{1}(g, \lambda(\cdot) ; t, z)$.

Proof. By Theorem 1 we have that

$$
\begin{aligned}
& \lambda^{m-1}\left(\partial_{z}^{1 / p}\right) u(t, z)=\left(\partial_{t}^{1 / p}\right)^{p-1} \int_{0}^{t} \sum_{n=m-1}^{\infty} \frac{(t-s)^{n / p}}{\Gamma(1+n / p)}\left(\begin{array}{c}
n \\
m-1
\end{array}\right) \lambda^{n}\left(\partial_{z}^{1 / p}\right) g(s, z) d s \\
= & \left(\partial_{t}^{1 / p}\right)^{p-1} \int_{0}^{t} \frac{1}{2 p \pi i} \oint_{|w|=\varepsilon}^{p} g(s, w) \int_{\zeta_{0}}^{\infty} e_{1 / p, m}\left((t-s) \lambda^{p}\left(\zeta^{1 / p}\right)\right) e_{1 / p}(z \zeta) e^{-w \zeta} d \zeta d w d s \\
= & \left(\partial_{t}^{1 / p}\right)^{p-1} \frac{1}{2 p \pi i} G_{m}(g, \lambda(\cdot) ; t, z) .
\end{aligned}
$$

Hence we obtain (a). To show (b) we take $k \in\{0, \ldots, m-1\}$ and $v(t, z)=\left(\partial_{t}^{1 / p}-\right.$ $\left.\lambda\left(\partial_{z}^{1 / p}\right)\right)^{k} u(t, z)$. Applying (a) we have

$$
\lambda^{m-1}\left(\partial_{z}^{1 / p}\right) v(t, z)=\left(\partial_{t}^{1 / p}\right)^{p-1} \frac{1}{2 p \pi i}\left(\partial_{t}^{1 / p}-\lambda\left(\partial_{z}^{1 / p}\right)\right)^{k} G_{m}(g, \lambda(\cdot) ; t, z) .
$$


On the other hand, $v(t, z)$ satisfies (20) with $m$ replaced by $m-k$. Hence by (a)

$$
\lambda^{m-1-k}\left(\partial_{z}^{1 / p}\right) v(t, z)=\left(\partial_{t}^{1 / p}\right)^{p-1} \frac{1}{2 p \pi i} G_{m-k}(g, \lambda(\cdot) ; t, z)
$$

Combining (23) and (24) we obtain (b). To show (c) we consider the Cauchy problem (20) with $m=1$. Then by (a) we have

$$
g(t, z)=\left(\partial_{t}^{1 / p}-\lambda\left(\partial_{z}^{1 / p}\right)\right) u(t, z)=\left(\partial_{t}^{1 / p}-\lambda\left(\partial_{z}^{1 / p}\right)\right)\left(\partial_{t}^{1 / p}\right)^{p-1} \frac{1}{2 p \pi i} G_{1}(g, \lambda(\cdot) ; t, z) .
$$

Combining Lemmas 1 and 2 we obtain

Proposition 9. Let us assume that $s>1, d \in \mathbb{R}$ and $\hat{u}(t, z)$ is a formal solution of (20). Then

$$
u(t, z) \in \mathcal{O}_{1 / p}^{s}\left(\hat{S}_{d} \times \hat{S}_{d+p \arg \lambda}\right) \Longleftrightarrow g(t, z) \in \mathcal{O}_{1 / p}^{s}\left(\hat{S}_{d} \times \hat{S}_{d+p \arg \lambda}\right) .
$$

By the above, we have the sufficient condition for (21)

$$
g(t, z) \in \mathcal{O}_{1 / p}^{s}\left(\hat{S}_{d} \times \hat{S}_{d+p \arg \lambda}\right) \Longrightarrow u(t, z) \in \mathcal{O}_{1 / p}^{s}\left(\hat{S}_{d} \times D\right),
$$

which unfortunately is too strong. Indeed, the function $u(t, z)=\frac{t}{1-z} \in \mathcal{O}\left(\hat{S}_{0} \times D\right)$ is a solution of

$$
\partial_{t} u-\partial_{z} u=\frac{1}{1-z}-\frac{t}{(1-z)^{2}}, \quad u(0, z)=0,
$$

but inhomogeneity is not analytic on $\hat{S}_{0} \times \hat{S}_{0}$.

From this we must find another condition on inhomogeneity, which characterise the solution of (20) satisfying (21). Such condition, unfortunately not so direct, is given by

Proposition 10. Let us assume that $s>1, d \in \mathbb{R}$ and $\hat{u}(t, z)$ is a formal solution of (20). Then

$$
u(t, z) \in \mathcal{O}_{1 / p}^{s}\left(\hat{S}_{d} \times D\right) \Longleftrightarrow G_{m}(g, \lambda(\cdot) ; t, z) \in \mathcal{O}_{1 / p}^{s}\left(\hat{S}_{d} \times D\right) .
$$

Proof. Immediately by Lemma 2 .

By Lemma 2, we have

Remark 4. If $G_{m}(g, \lambda(\cdot) ; t, z) \in \mathcal{O}_{1 / p}^{s}\left(\hat{S}_{d} \times D\right)$ then also $G_{j}(g, \lambda(\cdot) ; t, z) \in \mathcal{O}_{1 / p}^{s}\left(\hat{S}_{d} \times\right.$ $D)$ for $j=1, \ldots, m-1$ and $g(t, z) \in \mathcal{O}_{1 / p}^{s}\left(\hat{S}_{d} \times D\right)$.

Similarly, by Proposition 9 , we obtain

Remark 5. If $g(t, z) \in \mathcal{O}_{1 / p}^{s}\left(\hat{S}_{d} \times \hat{S}_{d+p \arg \lambda}\right)$ then $G_{m}\left(g, \lambda\left(\partial_{z}^{1 / p}\right) ; t, z\right) \in \mathcal{O}_{1 / p}^{s}\left(\hat{S}_{d} \times\right.$ $D)$.

As a corollary to Theorem 2, we have

Proposition 11. Let $\hat{u}(t, z)=\sum_{j=1}^{l} \sum_{k=0}^{m_{j}-1} \hat{u}_{j k}(t, z)$ be a decomposition of solution of (11) constructed in Theorem 2. Then for $n_{1}, n_{2} \in \mathbb{N}, s>1$ and $d_{1}, d_{2} \in \mathbb{R}$ we have that $u(t, z) \in \mathcal{O}_{n_{1} / p, n_{2} / p}^{s}\left(\hat{S}_{d_{1}} \times D\right)$ if and only if $u_{j k}(t, z) \in \mathcal{O}_{n_{1} / p, n_{2} / p}^{s}\left(\hat{S}_{d_{1}} \times D\right)$ for every $k=1, . ., m_{j}$ and $j=1, \ldots, l$. 
Proof. $(\Longleftarrow)$ If $u_{j k}(t, z) \in \mathcal{O}_{m / p, n / p}^{s}\left(\hat{S}_{d_{1}} \times D\right)$ for every $k=1, . ., m_{j}$ and $j=1, \ldots, l$ then also $\hat{u}(t, z)=\sum_{j=1}^{l} \sum_{k=1}^{m_{j}} \hat{u}_{j k}(t, z)$ satisfies the same property.

$(\Longrightarrow)$ We define for $k=1, . ., m_{j}$ and $j=1, \ldots, l$ the operator

$$
P_{j k}\left(\partial_{t}^{1 / p}, \partial_{z}^{1 / p}\right):=\left(\partial_{t}^{1 / p}-\lambda_{j}\left(\partial_{z}^{1 / p}\right)\right)^{m_{j}-k} \prod_{n=1, n \neq j}^{l}\left(\partial_{t}^{1 / p}-\lambda_{n}\left(\partial_{z}^{1 / p}\right)\right)^{m_{n}}
$$

Observe that $\tilde{u}_{j k}(t, z):=P_{j k}\left(\partial_{t}^{1 / p}, \partial_{z}^{1 / p}\right) u(t, z)$ satisfies the equation

$$
\left\{\begin{array}{l}
\left(\partial_{t}^{1 / p}-\lambda_{j}\left(\partial_{z}^{1 / p}\right)\right)^{k} \tilde{u}_{j k}(t, z)=\hat{g}(t, z) \\
\left(\partial_{t}^{1 / p}\right)^{n} \tilde{u}_{j k}(0, z)=0,
\end{array} n=0, \ldots, k-1 .\right.
$$

On the other hand, by Theorem $2, u_{j k}(t, z)$ satisfies

$$
\begin{cases}\left(\partial_{t}^{1 / p}-\lambda_{j}\left(\partial_{z}^{1 / p}\right)\right)^{k} u_{j k}(t, z)=\hat{g}_{j k}(t, z) & \\ \left(\partial_{t}^{1 / p}\right)^{n} u_{j k}(0, z)=0, & n=0, \ldots, k-1,\end{cases}
$$

where $\hat{g}_{j k}(t, z)=d_{j k}\left(\partial_{z}^{1 / p}\right) \hat{g}(t, z)$. Hence

$$
u_{j k}(t, z)=d_{j k}\left(\partial_{z}^{1 / p}\right) \tilde{u}_{j k}(t, z)=d_{j k}\left(\partial_{z}^{1 / p}\right) P_{j k}\left(\partial_{t}^{1 / p}, \partial_{z}^{1 / p}\right) u(t, z)
$$

and if $u(t, z) \in \mathcal{O}_{m / p, n / p}^{s}\left(\hat{S}_{d_{1}} \times D\right)$ then also $u_{j k}(t, z) \in \mathcal{O}_{m / p, n / p}^{s}\left(\hat{S}_{d_{1}} \times D\right)$.

\section{Summable solution}

If the pole order of $\lambda(\xi)$ is equal to $q>1$, we apply the operator $B^{1 / p, q / p}$ to the study of summability property. In this way we reduce the problem of summability to the study of the analytic solutions of the Kowalevskaya type equations considered in the previous section. As a result, we obtain the characterisation of summability of $\hat{u}(t, z)$ in terms of $\hat{g}(t, z)$.

Theorem 4. Let us assume that $\hat{u}(t, z)$ is a formal solution of the equation

$$
\left\{\begin{array}{l}
\left(\partial_{t}^{1 / p}-\lambda\left(\partial_{z}^{1 / p}\right)\right)^{m} u(t, z)=\hat{g}(t, z) \\
\left(\partial_{t}^{1 / p}\right)^{j} u(0, z)=0
\end{array} \quad j=0, \ldots, m-1,\right.
$$

a pole order of $\lambda(\xi)$ is equal to $q=\mu / \nu>1(\mu, \nu \in \mathbb{N})$ and $d \in \mathbb{R}$.

Then the following conditions are equivalent

(a) $\hat{u}(t, z)$ is $(q-1)^{-1}$-summable in a direction $d$,

(b) $B^{1 / p, q / p} \hat{u}(t, z) \in \mathcal{O}_{q / p, 1 / p}^{q /(q-1)}\left(\hat{S}_{(d+2 n \pi) / q} \times D\right)$ for $n=0, \ldots, \mu-1$,

(c) $G_{m}\left(B^{1 / p, q / p} \hat{g}, \tilde{\lambda}_{j}\left(\partial_{z}^{1 / \nu p}\right) ; t, z\right) \in \mathcal{O}_{q / p, 1 / p}^{q /(q-1)}\left(\hat{S}_{(d+2 n \pi) / q} \times D\right)$ for $n=0, \ldots, \mu-1$ and $j=0, \ldots, \mu-1$, where $\tilde{\lambda}_{j}(\xi):=e^{j 2 \pi i / \mu} \lambda^{1 / \mu}\left(\xi^{\nu}\right)$.

Proof. The equivalence between (a) and (b) is given by Proposition 7 . To show equivalence between (b) and (c), observe that $v(t, z):=B^{1 / p, q / p} \hat{u}(t, z)$ satisfies the equation

$$
\begin{cases}\left(\partial_{t}^{\mu / \nu p}-\lambda\left(\left(\partial_{z}^{1 / \nu p}\right)^{\nu}\right)\right)^{m} v(t, z)=\hat{h}(t, z) & \\ \left(\partial_{t}^{1 / \nu p}\right)^{j} v(0, z)=0, & j=0, \ldots, \mu m-1,\end{cases}
$$


where $\hat{h}(t, z)=B^{1 / p, q / p} \hat{g}(t, z)$. By Theorem $2, v(t, z)=\sum_{j=0}^{\mu-1} \sum_{k=1}^{m} v_{j k}(t, z)$ and $v_{j k}(t, z)$ satisfies the equation

$$
\left\{\begin{array}{l}
\left(\partial_{t}^{1 / \nu p}-\tilde{\lambda}_{j}\left(\partial_{z}^{1 / \nu p}\right)\right)^{k} v_{j k}(t, z)=\hat{h}_{j k}(t, z), \\
\left(\partial_{t}^{1 / \nu p}\right)^{n} v_{j k}(0, z)=0,
\end{array} \quad n=0, \ldots, k-1,\right.
$$

where $\hat{h}_{j k}(t, z)=d_{j k}\left(\partial_{z}^{1 / \nu p}\right) \hat{h}(t, z)$ for some holomorphic functions $d_{j k}(\xi)$ of polynomial growth and $\tilde{\lambda}_{j}(\xi):=e^{j 2 \pi i / \mu} \lambda^{1 / \mu}\left(\xi^{\nu}\right)$. Observe that a pole order of $\tilde{\lambda}_{j}(\xi)$ is equal to 1.

By Proposition 11, $v(t, z) \in \mathcal{O}_{q / p, 1 / p}^{q /(q-1)}\left(\hat{S}_{(d+2 n \pi) / q} \times D\right)$ if and only if $v_{j k}(t, z) \in$ $\mathcal{O}_{q / p, 1 / p}^{q /(q-1)}\left(\hat{S}_{(d+2 n \pi) / q} \times D\right)(n \in \mathbb{N})$ for $j=0, \ldots, \mu-1$ and $k=1, \ldots, m$. Moreover, by Proposition 10, $v_{j k}(t, z) \in \mathcal{O}_{q / p, 1 / p}^{q /(q-1)}\left(\hat{S}_{(d+2 n \pi) / q}\right)$ if and only if $G_{k}\left(h_{j k}, \tilde{\lambda}_{j}(\cdot) ; t, z\right) \in$ $\mathcal{O}_{q / p, 1 / p}^{q /(q-1)}\left(\hat{S}_{(d+2 n \pi) / q} \times D\right)$. Since $G_{k}\left(h_{j k}, \tilde{\lambda}_{j}(\cdot) ; t, z\right)=d_{j k}\left(\partial_{z}^{1 / \nu p}\right) G_{k}\left(h, \tilde{\lambda}_{j}(\cdot) ; t, z\right)$ and using Remark 4 we obtain the equivalence between (b) and (c).

Corollary 1. Under the assumptions of Theorem 4 , if moreover $B^{1 / p, q / p} \hat{g}(t, z) \in$ $\mathcal{O}_{q / p, 1 / p}^{q /(q-1)}\left(\hat{S}_{(d+2 k \pi) / q} \times \hat{S}_{(d+p \lambda+2 l \pi) / q}\right)$ for every $k=0, \ldots, \mu-1, l=0, \ldots, \mu-1$, where $\lambda$ is a leading term of $\lambda(\xi)$, then the formal solution $\hat{u}(t, z)$ of $(25)$ is $(q-1)^{-1}$. summable in a direction $d$.

Proof. Repeating the proof of Theorem 4 we have that

$$
B^{1 / p, \mu / \nu p} \hat{u}(t, z)=\sum_{j=0}^{\mu-1} \sum_{k=1}^{m} v_{j k}(t, z)
$$

and $v_{j k}(t, z)$ satisfies $(26)$. Observe that

$$
\lim _{\xi \rightarrow \infty} \frac{\tilde{\lambda}_{j}(\xi)}{\xi}=\lim _{\xi \rightarrow \infty} e^{2 j \pi i / \mu}\left(\frac{\lambda\left(\xi^{\nu}\right)}{\xi^{\mu}}\right)^{1 / \mu}=e^{2 j \pi i / \mu_{j}} \lambda^{1 / \mu}=: \tilde{\lambda}_{j}
$$

and $\arg \tilde{\lambda}_{j}=(\arg \lambda+2 j \pi) / \mu$.

Hence $B^{1 / p, q / p} \hat{g}(t, z) \in \mathcal{O}_{q / p, 1 / p}^{q /(q-1)}\left(\hat{S}_{(d+2 k \pi) / q} \times \hat{S}_{(d+2 k \pi) / q+p \nu \arg \tilde{\lambda}_{j}}\right)$ for $j, k=$ $0, \ldots, \mu-1$ if and only if $B^{1 / p, q / p} \hat{g}(t, z) \in \mathcal{O}_{q / p, 1 / p}^{q /(q-1)}\left(\hat{S}_{(d+2 k \pi) / q} \times \hat{S}_{(d+2 l \pi+\arg \lambda) / q}\right)$ $(k, l=0, \ldots, \mu-1)$. The conclusion is given by Remark 5 .

\section{Multisummable solution}

In this section we return to the general inhomogeneous equation

$$
\left\{\begin{array}{l}
P\left(\partial_{t}^{1 / p}, \partial_{z}^{1 / p}\right) u(t, z)=\hat{f}(t, z) \\
\left(\partial_{t}^{1 / p}\right)^{n} u(0, z)=0
\end{array} \quad \text { for } \quad n=0, \ldots, m-1,\right.
$$

where

$$
P(\lambda, \xi)=P_{0}(\xi) \prod_{j=1}^{\tilde{n}} \prod_{i=1}^{l_{j}}\left(\lambda-\lambda_{j i}(\xi)\right)^{m_{j i}}
$$

and $\lambda_{j i}(\xi)$ is a characteristic root of pole order $q_{j} \in \mathbb{Q}$ for $j=1, \ldots, \tilde{n}$ and $i=1, \ldots, l_{j}$. Without loss of generality we may assume that there exist exactly $n$ pole orders of characteristic roots, which are greater than 1 , say $1<q_{1}<\ldots<q_{n}<\infty$ and let $\mu_{j}, \nu_{j} \in \mathbb{N}$ and $k_{j}>0$ be such that $\mu_{j} / \nu_{j}=q_{j}$ and $k_{j}=\left(q_{i}-1\right)^{-1}$ for $j=1, \ldots, n$. 
By the Duhamel principle the formal solution $\hat{u}(t, z)$ is determined by a formal power series $\hat{g}(t, z) \in \mathbb{E}_{1 / p}\left[\left[t^{1 / p}\right]\right]$, which satisfies the equation $P_{0}\left(\partial_{z}\right) \hat{g}(t, z)=$ $\hat{f}(t, z)$.

By Theorem 2

$$
\hat{u}(t, z)=\sum_{j=1}^{\tilde{n}} \sum_{i=1}^{l_{j}} \sum_{m=1}^{m_{i j}} \hat{u}_{j i m}(t, z),
$$

where $\hat{u}_{j i m}(t, z)$ satisfies

$$
\left\{\begin{array}{l}
\left(\partial_{t}^{1 / p}-\lambda_{j i}\left(\partial_{z}^{1 / p}\right)\right)^{m} u_{j i m}(t, z)=\hat{g}_{j i m}(t, z)=d_{j i m}\left(\partial_{z}^{1 / p}\right) \hat{g}(t, z) \\
\left(\partial_{t}^{1 / p}\right)^{l} u_{j i m}(0, z)=0 \text { for } n=0, \ldots, m-1
\end{array}\right.
$$

To study of summability property of formal solution of (27), we define a kind of multisummability as follows

Definition 12. Let $\left(d_{1}, \ldots, d_{n}\right) \in \mathbb{R}^{n}$ be an admissible multidirection. We say that $\hat{u}(t, z)$ is $\left(k_{1}, \ldots, k_{n}\right)$-summable in the multidirection $\left(d_{1}, \ldots, d_{n}\right)$ with respect to decomposition (28) if and only if $\hat{u}_{j i m}(t, z)$ is $k_{j}$-summable in the direction $d_{j}$ (for $j=1, \ldots, n$ ) and is convergent (for $j=n+1, \ldots, \tilde{n}$ ), where $i=1, \ldots, l_{j}$ and $m=1, \ldots, m_{j i}$.

Remark 6. If $g(t, z) \in \mathcal{O}_{1 / p}\left(D^{2}\right)$ then, by Theorem $3, \hat{u}_{j i m}(t, z)$ is a Gevrey series of order $q_{j}-1$. Hence, if $\hat{u}(t, z)$ is $\left(k_{1}, \ldots, k_{n}\right)$-summable in the multidirection $\left(d_{1}, \ldots, d_{n}\right)$ then $\hat{u}_{j i m}(t, z)$ is $k_{j}$-summable in the direction $d_{j}$ (if $q_{j}>1$ ) or is convergent (if $\left.q_{j} \leq 1\right)$. It means that $\hat{u}(t, z)$ is $\left(k_{1}, \ldots, k_{n}\right)$-summable in the multidirection $\left(d_{1}, \ldots, d_{n}\right)$ with respect to decomposition $(28)$.

Observe that, by Theorem 4, we have

Theorem 5. Under the above conditions, the formal solution $\hat{u}(t, z)$ of (27) determined by $\hat{g}(t, z)$ is $\left(k_{1}, \ldots, k_{n}\right)$-summable in an admissible multidirection $\left(d_{1}, \ldots, d_{n}\right)$ with respect to decomposition (28) if and only if $G_{m_{j i}}\left(B^{1 / p, q_{j} / p} \hat{g}, \tilde{\lambda}_{j i k}\left(\partial_{z}^{1 / v_{j} p}\right) ; t, z\right) \in$ $\mathcal{O}_{q_{j} / p, 1 / p}^{k_{j}+1}\left(\hat{S}_{\left(d_{j}+2 l \pi\right) / q_{j}} \times D\right)$ for $l=0, \ldots, \mu_{j}-1 k=0, \ldots, \mu_{j}-1, i=1, \ldots, l_{j}$ and $j=1, \ldots, n$, where $\tilde{\lambda}_{j i k}(\xi):=e^{k 2 \pi i / \mu_{j}} \lambda_{j i}^{1 / \mu_{j}}\left(\xi^{\nu_{j}}\right)$.

Moreover, by Remark 6, we obtain

Corollary 2. Under the above conditions, the formal solution $\hat{u}(t, z)$ of (27) determined by $g(t, z) \in \mathcal{O}_{1 / p}\left(D^{2}\right)$ is $\left(k_{1}, \ldots, k_{n}\right)$-summable in an admissible multidirection $\left(d_{1}, \ldots, d_{n}\right)$ if and only if $G_{m_{j i}}\left(B^{1 / p, q_{j} / p} g, \tilde{\lambda}_{j i k}\left(\partial_{z}^{1 / v_{j} p}\right) ; t, z\right) \in \mathcal{O}_{q_{j} / p, 1 / p}^{k_{j}+1}\left(\hat{S}_{\left(d_{j}+2 l \pi\right) / q_{j}} \times\right.$ D) for $l=0, \ldots, \mu_{j}-1 k=0, \ldots, \mu_{j}-1, i=1, \ldots, l_{j}$ and $j=1, \ldots, n$, where $\tilde{\lambda}_{j i k}(\xi):=e^{k 2 \pi i / \mu_{j}} \lambda_{j i}^{1 / \mu_{j}}\left(\xi^{\nu_{j}}\right)$.

Repeating the previous considerations with Theorem 4 replaced by Corollary 1 , we also obtain the following sufficient condition for multisummability

Corollary 3. Under the above conditions, if $B^{1 / p, q_{j} / p} \hat{g}(t, z) \in \mathcal{O}_{q_{j} / p, 1 / p}^{k_{j}+1}\left(\hat{S}_{\left(d_{j}+2 k \pi\right) / q_{j}} \times\right.$ $\left.\hat{S}_{\left(d_{j}+p \lambda_{j i}+2 l \pi\right) / q_{j}}\right)$ for $k=0, \ldots, \mu_{j}-1, l=0, \ldots, \mu_{j}-1, i=1, \ldots, l_{j}$ and $j=1, \ldots, n$, then the formal solution $\hat{u}(t, z)$ of (27) determined by $\hat{g}(t, z)$ is $\left(k_{1}, \ldots, k_{n}\right)$-summable in the admissible multidirection $\left(d_{1}, \ldots, d_{n}\right)$ with respect to decomposition (28). 


\section{EXAMPLE}

In the last section we give a simple example, which illustrate this theory. Namely, let $\hat{u}(t, z)$ be a formal solution of

$$
\left(\partial_{t}-\partial_{z}^{2}\right)\left(\partial_{t}-\partial_{z}^{3}\right) u(t, z)=\hat{f}(t, z), \quad u(0, z)=\partial_{t} u(0, z)=0 .
$$

Then $\hat{u}(t, z)=\hat{u}_{1}(t, z)+\hat{u}_{2}(t, z)$, where

$$
\begin{aligned}
\left(\partial_{t}-\partial_{z}^{2}\right) u_{1}(t, z) & =c_{1}\left(\partial_{z}\right) \hat{f}(t, z)=: \hat{f}_{1}(t, z), \quad u_{1}(0, z)=0, \quad c_{1}(\zeta)=\frac{1}{\zeta^{2}(1-\zeta)} \\
\left(\partial_{t}-\partial_{z}^{3}\right) u_{2} & =c_{2}\left(\partial_{z}\right) \hat{f}(t, z)=: \hat{f}_{2}(t, z), \quad u_{1}(0, z)=0, \quad c_{2}(\zeta)=\frac{1}{\zeta^{2}(\zeta-1)} .
\end{aligned}
$$

Observe that $\lambda_{1}(\zeta)=\zeta^{2}$ and $\lambda_{2}(\zeta)=\zeta^{3}$, so $q_{1}=2$ and $q_{2}=3$.

Applying the operators $B^{1, q_{i}}$ we have that $v_{i}(t, s):=B^{1, q_{i}} \hat{u}_{i}(t, s)(i=1,2)$ satisfy

$$
\begin{array}{lll}
\left(\partial_{t}^{2}-\partial_{z}^{2}\right) v_{1}(t, z)=B^{1,2} \hat{f}_{1}(t, z)=: g_{1}(t, z), & \partial_{t}^{n} v_{1}(0, z)=0, & n=0,1 \\
\left(\partial_{t}^{3}-\partial_{z}^{3}\right) v_{2}(t, z)=B^{1,3} \hat{f}_{2}(t, z)=: g_{2}(t, z), & \partial_{t}^{n} v_{1}(0, z)=0, & n=0,1,2 .
\end{array}
$$

We have $v_{1}(t, z)=v_{11}(t, z)+v_{12}(t, z)$, where

$$
\begin{aligned}
& \left(\partial_{t}-\partial_{z}\right) v_{11}(t, z)=d_{11}\left(\partial_{z}\right) g_{1}(t, z)=: g_{11}(t, z), \quad v_{11}(0, z)=0, \quad d_{11}(\zeta)=\frac{1}{2 \zeta} \\
& \left(\partial_{t}+\partial_{z}\right) v_{12}(t, z)=d_{12}\left(\partial_{z}\right) \hat{g}_{1}(t, z)=: g_{12}(t, z), \quad v_{12}(0, z)=0, \quad d_{12}(\zeta)=-\frac{1}{2 \zeta} .
\end{aligned}
$$

It means that $v_{11}(t, z)=\int_{0}^{t} g_{11}(s, z+t-s) d s$ and $v_{12}(t, z)=\int_{0}^{t} g_{12}(s, z-(t-s)) d s$ Analogously $v_{2}(t, z)=v_{21}(t, z)+v_{22}(t, z)+v_{23}(t, z)$, where

$$
\begin{gathered}
\left(\partial_{t}-\partial_{z}\right) v_{21}(t, z)=d_{21}\left(\partial_{z}\right) g_{2}(t, z)=: g_{21}(t, z), \quad v_{21}(0, z)=0, \quad d_{21}(\zeta)=\frac{1}{3 \zeta^{2}}, \\
\left(\partial_{t}-e^{2 \pi i / 3} \partial_{z}\right) v_{22}=d_{22}\left(\partial_{z}\right) g_{2}(t, z)=: g_{22}(t, z), \quad v_{22}(0, z)=0, \quad d_{22}(\zeta)=\frac{e^{2 \pi i / 3}}{3 \zeta^{2}}, \\
\left(\partial_{t}-e^{4 \pi i / 3} \partial_{z}\right) v_{23}=d_{23}\left(\partial_{z}\right) g_{2}(t, z)=: g_{23}(t, z), \quad v_{23}(0, z)=0, \quad d_{23}(\zeta)=\frac{e^{4 \pi i / 3}}{3 \zeta^{2}}
\end{gathered}
$$

Hence $v_{21}(t, z)=\int_{0}^{t} g_{21}(s, z+t-s) d s, v_{22}(t, z)=\int_{0}^{t} g_{22}\left(s, z+e^{2 \pi i / 3}(t-s)\right) d s$ and $v_{23}(t, z)=\int_{0}^{t} g_{23}\left(s, z+e^{4 \pi i / 3}(t-s)\right) d s$.

Finally, we have that if $f(t, z) \in \mathcal{O}\left(D^{2}\right)$ then the formal solution $\hat{u}(t, z)$ is $(1,1 / 2)$ summable in a multidirection $\left(d_{1}, d_{2}\right)$ $\Longleftrightarrow v_{1}(t, z) \in \mathcal{O}^{2}\left(\left(\hat{S}_{d_{1} / 2} \cup \hat{S}_{d_{1} / 2+\pi}\right) \times D\right)$ and $v_{2}(t, z) \in \mathcal{O}^{3 / 2}\left(\left(\hat{S}_{d_{2} / 3} \cup \hat{S}_{d_{2} / 3+2 \pi / 3} \cup\right.\right.$ $\left.\left.\hat{S}_{d_{2} / 3+4 \pi / 3}\right) \times D\right)$

$\Longleftrightarrow$ the function $f(t, z)$ satisfies the following conditions:

a) $\int_{0}^{t} B^{1,2} f(s, z+t-s) d s \in \mathcal{O}^{2}\left(\left(\hat{S}_{d_{1} / 2} \cup \hat{S}_{d_{1} / 2+\pi}\right) \times D\right)$,

b) $\int_{0}^{t} B^{1,2} f(s, z-(t-s)) d s \in \mathcal{O}^{2}\left(\left(\hat{S}_{d_{1} / 2} \cup \hat{S}_{d_{1} / 2+\pi}\right) \times D\right)$,

c) $\int_{0}^{t} B^{1,3} f(s, z+t-s) d s \in \mathcal{O}^{3 / 2}\left(\left(\hat{S}_{d_{2} / 3} \cup \hat{S}_{d_{2} / 3+2 \pi / 3} \cup \hat{S}_{d_{2} / 3+4 \pi / 3}\right) \times D\right)$,

d) $\int_{0}^{t} B^{1,3} f\left(s, z+e^{2 \pi i / 3}(t-s)\right) d s \in \mathcal{O}^{3 / 2}\left(\left(\hat{S}_{d_{2} / 3} \cup \hat{S}_{d_{2} / 3+2 \pi / 3} \cup \hat{S}_{d_{2} / 3+4 \pi / 3}\right) \times D\right)$,

e) $\int_{0}^{t} B^{1,3} f\left(s, z+e^{4 \pi i / 3}(t-s)\right) d s \in \mathcal{O}^{3 / 2}\left(\left(\hat{S}_{d_{2} / 3} \cup \hat{S}_{d_{2} / 3+2 \pi / 3} \cup \hat{S}_{d_{2} / 3+4 \pi / 3}\right) \times D\right)$. 


\section{REFERENCES}

1. W. Balser, Divergent solutions of the heat equation: on an article of Lutz, Miyake and Schäfke, Pacific J. of Math. 188 (1999), 53-63.

2. __ Formal power series and linear systems of meromorphic ordinary differential equations, Springer-Verlag, New York, 2000.

3. __ Multisummability of formal power series solutions of partial differential equations with constant coefficients, J. Differential Equations 201 (2004), 63-74.

4. __ Summability of formal power-series solutions of partial differential equations with constant coefficients, Journal of Mathematical Sciences 124 (2004), no. 4, 5085-5097.

5. _ Power series solutions of the inhomogeneous heat equation, Recent trends in Microlocal Analysis, Kôkyûroku RIMS, vol. 1412, 2005, pp. 151-159.

6. W. Balser, A. Duval, and S. Malek, Summability of formal solutions for abstract Cauchy problems and related convolution equations, Ulmer Seminare 11 (2006), 29-44.

7. W. Balser and M. Loday-Richaud, Summability of solutions of the heat equation with inhomogeneous thermal conductivity in two variables, Adv. Dyn. Syst. Appl. 4 (2009), 159-177.

8. W. Balser and M. Miyake, Summability of formal solutions of certain partial differential equations, Acta Sci. Math. (Szeged) 65 (1999), 543-551.

9. W. Balser and M. Yoshino, Gevrey order of formal power series solutions of inhomogeneous partial differential equations with constant coefficients, Funk. Ekvac. 53 (2010), 411-434.

10. K. Ichinobe, Integral representation for Borel sum of divergent solution to a certain nonKovalevski type equation, Publ. RIMS, Kyoto Univ. 39 (2003), 657-693.

11. A. Kilbas, Partial fractional differential equations and some of their applications, Analysis, Munchen 30 (2010), no. 1, 35-66.

12. A. Kilbas, M. Rivero, L. Rodriguez-Germa, and J. Trujillo, $\alpha$-Analytic solutions of some linear fractional differential equations with variable coefficients, Appl. Math. Comput. 187 (2007), 239-249.

13. A. Kilbas, H. Srivastava, and J. Trujillo, Theory and applications of fractional differential equations, North-Holland Mathematics Studies, vol. 204, Elsevier Science B.V., Amsterdam, 2006.

14. A. Kochubei, Fractional differential equations: $\alpha$-entire solutions, regular and irregular singularities, Fract. Calc. Appl. Anal. 12 (2009), no. 2, 135-158.

15. D.A. Lutz, M. Miyake, and R. Schäfke, On the Borel summability of divergent solutions of the heat equation, Nagoya Math. J. 154 (1999), 1-29.

16. S. Malek, On the summability of formal solutions of linear partial differential equations, J. Dyn. Control Syst. 11 (2005), no. 3, 389-403.

17. S. Michalik, Summability of divergent solutions of the n-dimensional heat equation, J. Differential Equations 229 (2006), 353-366.

18. _ Summability of formal solutions to the n-dimensional inhomogeneous heat equation, J. Math. Anal. Appl. 347 (2008), 323-332.

19. with constant coefficients, J. Differential Equations 249 (2010), 551-570.

20. _ Summability and fractional linear partial differential equations, J. Dyn. Control Syst. 16 (2010), no. 4, 557-584.

21. M. Miyake, Borel summability of divergent solutions of the Cauchy problem to nonKovaleskian equations, Partial Differential Equations and Their Applications, 1999, pp. 225239.

22. G. Sansone and J. Gerretsen, Lectures on the theory of functions of a complex variable, P. Noordhoff, Groningen, 1960.

23. S. Umarov and E. Saidamatov, A fractional analog of the Duhamel principle, Fract. Calc. Appl. Anal. 9 (2006), no. 1, 57-70.

Faculty of Mathematics and Natural Sciences, College of Science, Cardinal Stefan Wyszyński University, Wóycickiego 1/3, 01-938 WarszaWa, Poland

E-mail address: s.michalik@uksw.edu.pl

$U R L$ : www.impan.gov.pl/ slawek 